\title{
Precommitment low-level Neurog3 expression defines a long-lived mitotic endocrine-biased progenitor pool that drives production of endocrine-committed cells
}

\author{
Matthew E. Bechard, ${ }^{1}$ Eric D. Bankaitis, ${ }^{1}$ Susan B. Hipkens, ${ }^{1,2}$ Alessandro Ustione,${ }^{2,3}$ David W. Piston, ${ }^{2,3}$ \\ Yu-Ping Yang, ${ }^{1}$ Mark A. Magnuson, ${ }^{1,2}$ and Christopher V.E. Wright ${ }^{1}$ \\ ${ }^{1}$ Vanderbilt University Program in Developmental Biology, Department of Cell and Developmental Biology, Vanderbilt Center for \\ Stem Cell Biology, Vanderbilt University School of Medicine, Nashville, Tennessee 37232, USA; ${ }^{2}$ Department of Molecular \\ Physiology and Biophysics, Vanderbilt University School of Medicine, Nashville, Tennessee 37232, USA
}

The current model for endocrine cell specification in the pancreas invokes high-level production of the transcription factor Neurogenin 3 (Neurog3) in Sox $9^{+}$bipotent epithelial cells as the trigger for endocrine commitment, cell cycle exit, and rapid delamination toward proto-islet clusters. This model posits a transient Neurog3 expression state and short epithelial residence period. We show, however, that a Neurog $3^{\text {TA.LO }}$ cell population, defined as Neurog3 transcriptionally active and Sox $9^{+}$and often containing nonimmunodetectable Neurog3 protein, has a relatively high mitotic index and prolonged epithelial residency. We propose that this endocrine-biased mitotic progenitor state is functionally separated from a pro-ductal pool and endows them with long-term capacity to make endocrine fate-directed progeny. A novel BAC transgenic Neurog3 reporter detected two types of mitotic behavior in Sox ${ }^{+}$ Neurog $3^{\text {TA.LO }}$ progenitors, associated with progenitor pool maintenance or derivation of endocrine-committed Neurog $3{ }^{\mathrm{HI}}$ cells, respectively. Moreover, limiting Neurog3 expression dramatically increased the proportional representation of Sox $9^{+}$Neurog $3^{\text {TA.LO }}$ progenitors, with a doubling of its mitotic index relative to normal Neurog3 expression, suggesting that low Neurog3 expression is a defining feature of this cycling endocrine-biased state. We propose that Sox $9^{+}$Neurog $3^{\text {TA.LO }}$ endocrine-biased progenitors feed production of Neurog $3^{\mathrm{HI}}$ endocrine-committed cells during pancreas organogenesis.

[Keywords: Neurog3; progenitor; endocrine-biased; mitotic]

Supplemental material is available for this article.

Received May 27, 2016; revised version accepted August 3, 2016.

During mammalian organogenesis, lineage specification and commitment involve passage through distinct progenitor/precursor states that rely on different combinations and levels of transcription factors (Wilkinson et al. 2013; Cano et al. 2014). In the current model of pancreatic endocrine cell formation, Neurogenin3 (Neurog3) expression in the epithelium rapidly progresses to a high-level production of protein (Neurog $3^{\mathrm{HI}}$ ) that leads to endocrine fate commitment, cell cycle exit, and delamination toward proto-islet clusters. Meta-analysis of published literature (see below), however, is suggestive of a broader pattern of lower-level Neurog3 expression across the epithelium that is substantially more prevalent than the actively delaminating endocrine-committed Neurog $3^{\mathrm{HI}}$

\footnotetext{
${ }^{3}$ Present address: Cell Biology and Physiology, Washington University School of Medicine, St. Louis, MO 63110, USA. Corresponding author: chris.wright@vanderbilt.edu

Article published online ahead of print. Article and publication date are online at http://www.genesdev.org/cgi/doi/10.1101/gad.284729.116.
}

population. The present study is focused on determining whether this low-Neurog3-expressing subpopulation represents the early-phase expression in post-mitotic cells on their way to becoming Neurog $3^{\mathrm{HI}}$ cells or endocrine fate-biased but uncommitted mitotic cells that have self-maintaining progenitor characteristics.

Pancreas organogenesis is divided into a primary transition and secondary transition (Pan and Wright 2011). During the primary transition (embryonic day 9.5 [E9.5] to E12.5), multipotent progenitor cells undergo apical polarization, forming microlumens that then coalesce to generate an epithelial plexus. Within this epithelial plexus, progenitor cells (around E12.5) segregate into "tip" and "trunk" domains. In the short-term, tip domains

\footnotetext{
(C) 2016 Bechard et al. This article is distributed exclusively by Cold Spring Harbor Laboratory Press for the first six months after the full-issue publication date (see http://genesdev.cshlp.org/site/misc/terms.xhtml). After six months, it is available under a Creative Commons License (Attribution-NonCommercial 4.0 International), as described at http://creativecommons.org/licenses/by-nc/4.0/.
} 
transiently retain their multipotency to form acinar, duct, and endocrine lineages before transitioning to a proacinar state. The trunk domain, a primary component of the epithelial plexus, consists of a pool of duct/endocrine bipotential Sox $9^{+} \mathrm{Hnflb}^{+} \mathrm{Nkx} 6.1^{+}$cells (Seymour et al. 2007; Solar et al. 2009). Recently, we reported that distinct modes of epithelial morphogenesis in the peripheral versus core regions of the developing pancreas work in concert to generate the final "epithelial tree" of the mature pancreas (Bankaitis et al. 2015). In the core, the epithelial plexus remodels into mature ductal tissues via growth and expansion (E12.5-E15.5) followed by plexus-to-duct transformation (E16.5-E18.5) (Bankaitis et al. 2015). Although the plexus is progressively lost, remodeling into "mature" ducts and ductal branches, it is present during all stages of the secondary transition. Even at late gestation, plexus-state epithelium generates as many endocrine cells as earlier in the secondary transition, suggesting that the plexus represents a primary endocrine progenitor niche (Bankaitis et al. 2015). Within the trunk plexus-state epithelium, the transcription factor Neurog3 is a key trigger factor for endocrine lineage commitment. Expressed in a subset of the $\mathrm{Sox}^{+}$duct/endocrine bipotent progenitors, high Neurog3 protein levels activate the expression of general proendocrine transcription factor genes (NeuroD1, Insm1A, and Is11) followed by those for endocrine lineage-specific factors (Pax4, Nkx2.2, and Arx). Neurog $3^{\text {NULL }}$ mice show a vast deficiency in endocrine cell production and a near absence at birth (Gradwohl et al. 2000; Johansson et al. 2007). An inducible Neurog3 overexpression system showed an endoderm-autonomous competence for Neurog $3^{\mathrm{HI}}$ cells to produce mostly a (glucagon-producing) cells in early pancreas organogenesis (E8.75-E12.5), with the ability to produce $\beta$ cells (and other endocrine cells in lesser number) arising in the secondary transition (Johansson et al. 2007). A large-scale clonal analysis demonstrated that the majority of Neurog $3^{\mathrm{HI}}$ cells each represents a unipotent precursor of a single hormone-expressing endocrine cell type (Desgraz and Herrera 2009); for example, insulin or glucagon. These studies show that the epithelial competence to produce the various endocrine hormone-secreting cells switches over time and that Neurog3 is necessary and sufficient for endocrine lineage commitment.

The transition from Neurog3 activation to an endocrine-committed Neurog ${ }^{\mathrm{HI}}$ state is thought to occur rapidly, predicting a low epithelial residency period for Neurog3-expressing cells. Our closer examination of the primary data in several studies, however, revealed evidence that could be interpreted as opposing this view. First, low-level Neurog3 expression within the secondary transition pancreatic epithelium seems widespread. Either Neurog $3^{\text {BAC.Cre }} ; R 26 R$ lineage tracing or a Neurog $3^{\mathrm{EGFP} /+}$ knock-in reporter detects a large number of intraepithelial cells that potentially represent cells in a Neurog3-expressing pre-endocrine-committed state (Zhang et al. 2009; Wang et al. 2010; Bankaitis et al. 2015). In the study by Villasenor et al. (2008), Neurog3 mRNA detection also suggests a Neurog3-expressing epithelial population that is broader than that visualized by Neurog3 immunodetection. Furthermore, in situ RNA hybridization with a cocktail of endocrine-specific probes, including Neurog3, showed a substantial occupancy of the epithelial trunk domain by putative endocrine progenitors or precursors (Zhou et al. 2007). Multiple studies report a small but significant number of nonendocrine cells as generated from Neurog3-expressing epithelial cells, suggesting that, in a minority of cases, activating Neurog3 expression does not unequivocally instruct endocrine commitment (Schonhoff et al. 2004; Wang et al. 2010). We propose that these studies suggest an intraepithelial subpopulation of Neurog3-expressing cells of significant interest in that it might represent a distinct, possibly mitotic, endocrine-biased progenitor state localized to or highly enriched within plexus-state epithelium, which feeds production of endocrine-committed cells during the secondary transition.

These studies prompted our investigation of this endocrine-biased Neurog3-expressing epithelial population, focusing on the hypothesis that it does represent a mitotic feeder population. Moreover, we also report a novel Neurog3 BAC transgenic reporter sensitive enough to mark low-level Neurog3-expressing cells, allowing a deeper analysis of endocrine specification and commitment. We present several lines of evidence that Sox9-expressing, Neurog3 transcriptionally active (Neurog $3^{\mathrm{TA}}$ ) cells with low or even immunologically undetectable Neurog3 protein (referred to here as Neurog $3^{\text {TA.LO }}$ cells) in fact do represent a mitotic progenitor state that is distinguishable from endocrine-committed Neurog $3^{\mathrm{HI}}$ cells and Sox $9^{+}$ Neurog $3^{-}$pro-duct progenitors. Our real-time analysis shows that Sox $9^{+}$Neurog $3^{\text {TA.LO }}$ progenitors undergo divisions that either maintain the Neurog $3^{\text {TA.LO }}$ progenitor pool or generate endocrine-committed Neurog $3^{\mathrm{HI}}$ cells. Furthermore, genetically limiting Neurog3 expression increased the proliferation and significantly expanded the proportional representation of the Sox $9^{+}$Neurog $3^{\text {TA.LO }}$ population. Our findings support a new model in which endocrine specification is driven by this novel Sox $9^{+} \mathrm{Neu}$ rog3 ${ }^{\text {TA.LO }}$ endocrine-biased progenitor pool, significantly advancing our understanding of endocrine cell ontogeny. We discuss potential parallels with the Neurog2-based regulation of the neural progenitor state versus neural specification. We propose that identifying the Neurog $3^{\text {TA.LO }}$ condition should focus attention on this state as being where intercellular signaling and intrinsic gene regulatory networks converge to regulate Neurog3 expression, control the balance between progenitor maintenance and endocrine specification/commitment, and perhaps identify an early intraepithelial stage at which hormone-specific lineage allocation might occur.

\section{Results}

Mitotic Sox $9^{+}$Neurog3 protein-low cells are maintained throughout the secondary transition

To study the initial phases of Neurog3 expression, we examined Neurog3 protein levels in cells located within or outside the epithelium at E12.5, E14.5, and E16.5. Using Sox 9 and Muc1 to mark epithelial cells and their lumenal 
surface, respectively, we observed two discernable states of Neurog3 expression: a Neurog3 protein-low (Neurog $3^{\mathrm{pLO}} / \mathrm{Sox}^{+} \mathrm{Mucl}^{+}$state and a Neurog3 protein-high $\left(\right.$ Neurog $3^{\mathrm{pHI}}$ ) state that is largely Sox $9^{-}$and $\mathrm{Muc1}^{-}$(Fig. 1A,B). Because we describe several distinct Neurog3positive populations, for clarity, we list the nomenclature used for each and the associated cell state in Supplemental Table S1. Comparing the Neurog3 immunofluorescence signal intensities showed that Neurog3 protein levels in epithelial Sox $9^{+} \mathrm{Mucl}^{+}$Neurog3 protein-positive (Neurog $3^{\text {Protein+ }}$ ) cells were fivefold lower than Neurog3 protein levels in delaminated Sox $9^{-} \mathrm{Muc1}^{-}$Neurog3 ${ }^{\text {Protein+ }}$ cells at E12.5 and threefold lower for the same comparison at E14.5 (Fig. 1C). Although an avidin-biotin amplification method was used to detect Neurog3, similar results were observed with nonamplifying indirect immunofluorescence (data not shown). The certainty of the Neurog3 low-level protein signal was determined by side-by-side comparison of wild-type and Neurog $3^{\text {null }}$ tissue (with no signal observed in null tissue) (Supplemental Fig. S1, A vs. B) and location over $\mathrm{DAPI}^{+}$nuclei. To evaluate the possible progenitor to precursor relationship between the Sox $9^{+}$Neurog $3^{\mathrm{pLO}}$ and Sox $9^{-}$Neurog $3^{\mathrm{pHI}}$ populations, respectively, their proportional representations with respect to each other were quantified at E12.5, E14.5, and E16.5 (Fig. 1D). One would expect the number of Neurog $3^{\mathrm{pLO}}$ relative to Neurog $3^{\mathrm{pHI}}$ cells to be largely equal during the secondary transition if Sox $9^{+}$Neurog $3^{\mathrm{pLO}}$ cells represented the early-stage upsweep of expression during the rapid progression from Neurog3 activation to Neu$\operatorname{rog} 3^{\mathrm{pHI}}$ in each endocrine-committing cell. At E12.5, however, Sox $9^{+}$Neurog $3^{\mathrm{pLO}}$ cells outnumbered Neurog $3^{\mathrm{pHI}}$ cells by a small but significant margin (Fig. 1D), while stages after E12.5 exhibited a shift in favor of Sox $9^{-}$Neurog $3^{\mathrm{pHI}}$ and away from Sox $9^{+}$Neurog $3^{\mathrm{pLO}}$ cells (Fig. 1D). These data are consistent with Sox $9^{+}$Neurog $3^{\text {pLO }}$ cells functioning as a progenitor pool that gives rise to Neu$\operatorname{rog} 3^{\mathrm{pHI}}$ endocrine precursors. To determine whether the Sox $9^{+}$Neurog $3^{\mathrm{pLO}}$ population varied during the secondary transition, their number as a proportion of the entire Sox $9^{+}$epithelium was quantified between E12.5 and E16.5. Despite a decrease from E12.5 to E14.5, the Sox $9^{+}$ Neurog $3^{\mathrm{pLO}}$ population remained at a similar level between E14.5 and E16.5 (Supplemental Fig. S2). Although the numbers of Sox $9^{+}$Neurog $3^{\mathrm{pLO}}$ cells seem quite low if scored relative to the entire epithelium, their representation is likely much higher within the central epithelial plexus (further supporting evidence is presented below), in which the majority of Neurog $3^{+}$cells reside and which produces endocrine cells with a relatively constant yield between E14.5 and E16.5 (Bankaitis et al. 2015). Because we suspected that the Sox $9^{+}$Neurog $3^{\mathrm{pLO}}$ pool functions as a cycling progenitor population, we tested its proliferative index relative to endocrine-committed Neurog $3^{\mathrm{pHI}}$ cells. Detecting phospho-histone $\mathrm{H} 3$ (pHH3) to mark cell cycle $\mathrm{M}$ phase, over $\mathrm{DAPI}^{+}$nuclei, substantial numbers of intraepithelial $\mathrm{pHH}^{+}{ }^{+}$Neurog $3^{\mathrm{pLO}}$ cells were found at E12.5 and E14.5 (Fig. 1E-H). Although, above, we used Sox 9 to mark intraepithelial cells, the fact that both Sox 9 and $\mathrm{pHH} 3$ antibodies were raised in rabbits prompted the use of Mucl (an apical surface marker) and DAPI to identify epithelial cells with a definitive apical lumen-contacting surface. Quantification revealed $11.5 \% \pm 1.7 \%$ of $\mathrm{Mucl}^{+} \mathrm{Neurog}^{\mathrm{pLO}}$ cells as $\mathrm{pHH}^{+}$at E12.5 versus $7.6 \% \pm 1.8 \%$ at E14.5 (Fig. 1I). Consistent with previous studies (Gu et al. 2002; Desgraz and Herrera 2009), Sox $9^{-}$Neurog3 $3^{\mathrm{pHI}}$ cells had very few $\mathrm{pHH}^{+}$cells and thus were largely post-mitotic (Fig. 1I). Quantifying immunofluorescence signal intensities in $\mathrm{Mucl}^{+} \mathrm{Neu}-$ rog3 ${ }^{\text {Protein }+} \mathrm{pHH}^{+}$cells versus $\mathrm{Muc1}^{-}$Neurog3 ${ }^{\text {Protein+ }}$ $\mathrm{pHH}^{-}$cells at E12.5 and E14.5 confirmed that intraepithelial $\mathrm{pHH}^{+}$Neurog3 $3^{\text {Protein }+}$ cells were in the Neurog $3^{\mathrm{pLO}}$ state (Fig. 1J). These data together support the idea that the intraepithelial Neurog $3^{\mathrm{pLO}}$ condition is an appreciably mitotic progenitor state that, as a population, perdures during the secondary transition. We postulate that the greater number and mitotic index of intraepithelial Neurog $3^{\mathrm{pLO}}$ cells at E12.5 (Fig. 1D,I) are possibly connected to the expansion of the plexus niche as the pancreatic epithelium enters the secondary transition period (Bankaitis et al. 2015).

Neurog3 transcriptional activity marks a mitotic Sox $9^{+}$ Neurog $3^{+}$endocrine progenitor population

As discussed above, previous reports showed Neurog3 mRNA expression to be broader than Neurog3 protein and to include intraepithelial cells. To investigate intraepithelial Neurog3-expressing cells further, we used the Neurog3 ${ }^{\text {BAC.Cre }}$ transgene (Schonhoff et al. 2004) to drive $R 26 R^{\text {EYFP }}$ recombination to mark Neurog3 ${ }^{\text {TA }}$ cells. Analysis at E14.5 revealed a heterogeneous pattern of EYFPlabeled Sox $9^{+}$Neurog $3^{\text {TA }}$ cells marked for having entered a Neurog3-expressing state (Supplemental Fig. S3). In peripheral regions of the pancreas, the proportion of EYFP-labeled Sox $9^{+}$Neurog3 ${ }^{\mathrm{TA}}$ intraepithelial cells was as low as $5 \%-8 \%$, while in central plexus-state regions, the proportional representation was locally as high as 54\% (Supplemental Fig. S3). These results are consistent with previous work showing that Neurog3-expressing cells are mostly derived from the central plexus state and much less from peripheral regions comprising mostly duct and ductal branches (Bankaitis et al. 2015). Quantification of central plexus regions showed an overall proportional representation of $24 \% \pm 2.4 \%(N=5)$ for EYFPlabeled Sox $9^{+}$Neurog $3^{\mathrm{TA}}$ cells among all Sox $9^{+}$cells. Endocrine commitment is thought to occur rapidly after Neurog3 activation, triggering Sox9 down-regulation, Neurog3 up-regulation, and epithelial delamination. However, within the Sox $9^{+}$EYFP-labeled Neurog $3^{\text {TA }}$ population, we consistently identified a number of scattered cells with a higher EYFP signal (Supplemental Fig. S3). We speculate that, following activation of Neurog3 expression, some Sox $9^{+}$Neurog $3^{\mathrm{TA}}$ cells remain intraepithelial for a prolonged period before moving to commitment delamination and thus accumulate more EYFP. Notably, the majority of EYFP-labeled Sox $9^{+}$Neurog $3^{\text {TA }}$ cells within these plexus regions were clustered and not fully interspersed with EYFP-negative cells. We propose that it is highly unlikely that all of these clustered cells are 


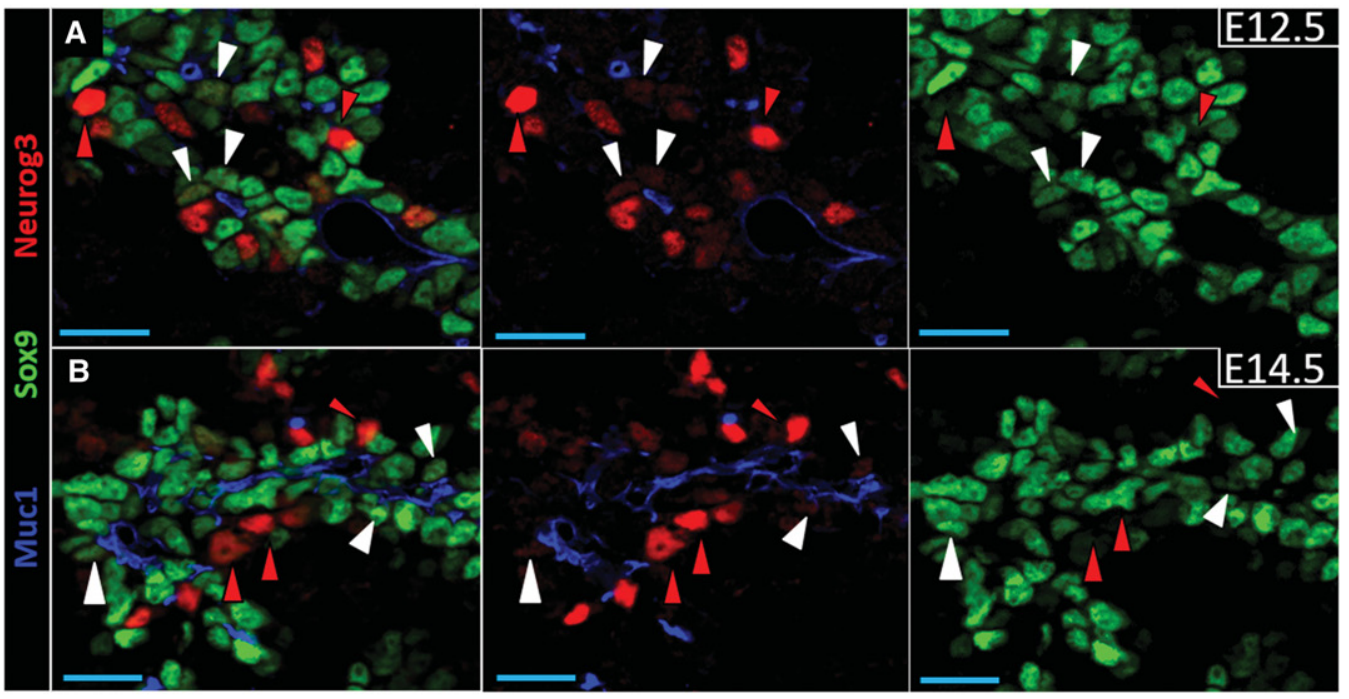

C
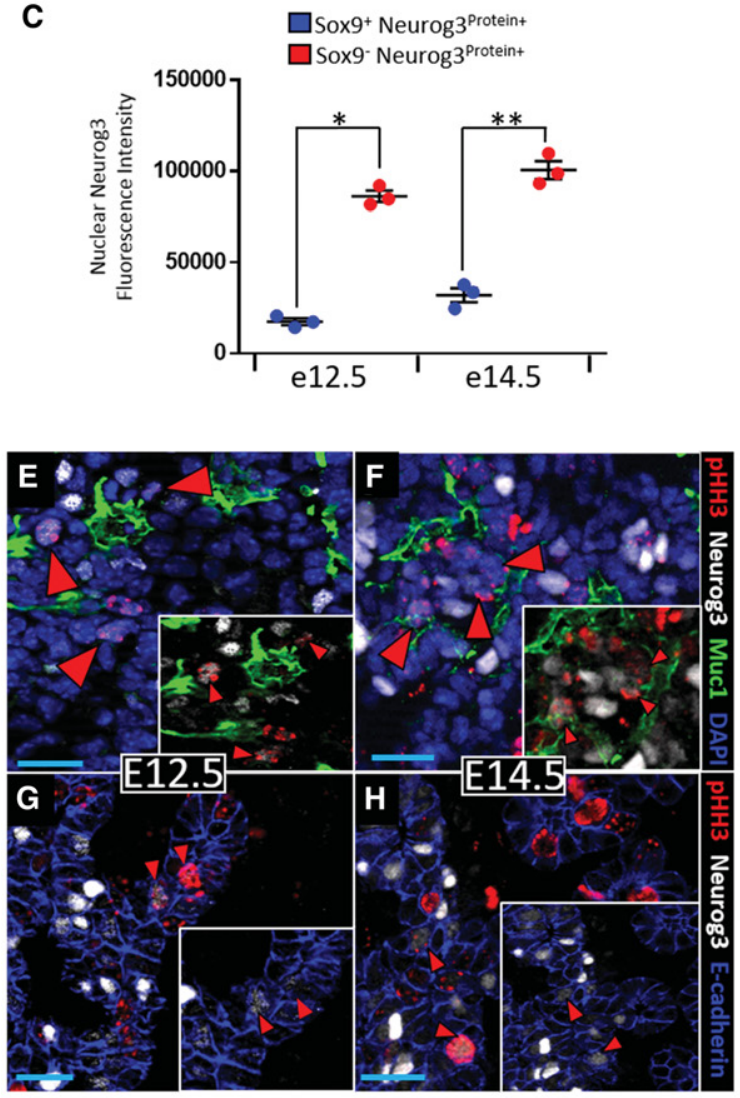

D

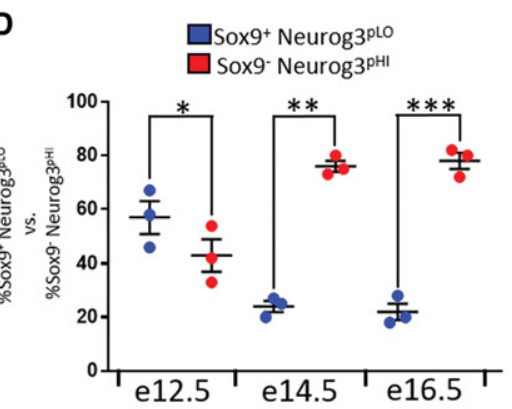

I

\begin{tabular}{|c|c|c|}
\hline & 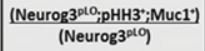 & 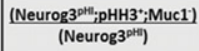 \\
\hline $\begin{array}{l}\mathrm{E} 12.5 \\
(\mathrm{~N}=4)\end{array}$ & $11.5 \% \pm 1.7 \%$ & $1.4 \% \pm 0.27 \%$ \\
\hline $\begin{array}{l}\mathrm{E} 14.5 \\
(\mathrm{~N}=3)\end{array}$ & $7.6 \% \pm 1.8 \%$ & $0.9 \% \pm 0.31 \%$ \\
\hline
\end{tabular}

$\mathbf{J}$

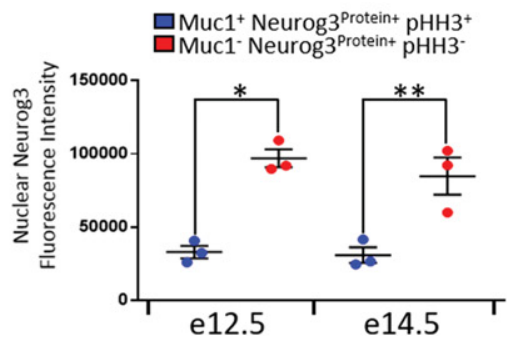

Figure 1. The Neurog $3^{\text {Protein+ }}$ population is comprised of mitotic Neurog $3^{\mathrm{pLO}}$ and post-mitotic Neurog $3^{\mathrm{pHI}}$ cells. E12.5 $(A)$ and E14.5 $(B)$ pancreatic epithelium showing Muc1, Sox 9 , and Neurog3. White and red arrowheads indicate Sox $9^{+}$Muc $1^{+} \mathrm{Neurog} 3^{\mathrm{pLO}}$ cells and Sox $9^{-}$ Muc1 $^{-}$Neurog3 ${ }^{\mathrm{pHI}}$, respectively. (C) Average fluorescence intensity of nuclear Neurog3 signal in Sox $9^{+}$Neurog $3^{\text {Protein+ }}{ }^{\text {versus Sox }} 9^{-}$Neurog $3^{\text {Protein }+}$ cells. $n=9$ cryosections; $N=3$ pancreata at E12.5 and E14.5. $\left({ }^{*}\right) P=2 \times 10^{-5} ;\left(^{* *}\right) P=0.0002 .(D)$ Percentage of Sox $9^{+}$Neurog $3^{\mathrm{pLO}}$ versus Sox9- Neurog $3^{\mathrm{pHI}}$ cells at E12.5 $(n=1114 ; N=3)$, E14.5 $(n=3797 ; N=3)$, and E16.5 $(n=4374 ; N=3) .\left(^{*}\right) P=0.0895 ;\left(^{* *}\right) P=3 \times 10^{-5}$; $\left(^{* * *}\right) P=0.0001$. E12.5 $(E, G)$ and E14.5 $(F, H)$ pancreatic epithelium showing pHH3, Neurog3, Muc1, and DAPI or pHH3, Neurog3, and E-

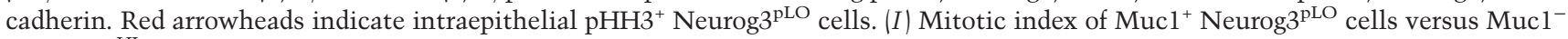
Neurog $3^{\mathrm{pHI}}$ cells at E12.5 $(n=1546)$ and E14.5 $(n=10080)$. (J) Average fluorescence intensity of nuclear Neurog3 signal in Muc1 ${ }^{+} \mathrm{Neu}^{-}$ $\operatorname{rog} 3^{\text {Protein+ }} \mathrm{pHH} 3^{+}$cells versus $\mathrm{Muc}^{-}{ }^{-}$Neurog $3^{\text {Protein+ }}{ }^{\mathrm{pHH}} 3^{-}$cells at E12.5 and E14.5, calculated as in $C$. Data are mean $\left.\pm \mathrm{SEM} .{ }^{*}\right) P=$ $\left.0.0005 ;{ }^{* *}\right) P=0.0087$. 
concurrently transitioning to the Neurog $3^{\mathrm{HI}}$ state. This observation thus supports the notion that low-level Neurog3 activation in Sox $9^{+}$cells, combined with a substantial residence period, leads to a sizeable intraepithelial representation of this cell population. It should be remembered that there is eventually only a tiny final contribution of EYFP-labeled Neurog $3^{\text {BAC.Cre }}$ traced cells to mature duct fates ( 1\%) (Schonhoff et al. 2004), meaning that essentially all of these "transiently intraepithelial" cells do eventually progress to endocrine commitment.

Within these EYFP-labeled Sox $9^{+}$Neurog $3^{\text {TA }}$ cells, in addition to some containing detectable but low-level Neurog3 protein (Neurog3 ${ }^{\text {TA.pLO}}$ ), many more had none detectable (Neurog3 ${ }^{\text {TA.pUD }}$ ) (Fig. 2A,B; Supplemental Table S1). The proliferation marker $\mathrm{pHH} 3$ revealed numerous mitotic intraepithelial Neurog $3^{\text {TA.pLO }}$ (defined here as $\mathrm{Mucl}^{+}$lumen-contacting) (presumably equivalent to those in Fig. 1E-H) as well as Neurog3 ${ }^{\text {TA.pUD }}$ cells (Fig. $2 \mathrm{C}, \mathrm{D})$. These data, along with the knowledge that nearly all ( 96\%) Neurog3-based lineage-labeled cells ultimately adopt the endocrine lineage (Schonhoff et al. 2004; Zhang et al. 2009), indicate that the Sox $9^{+}$Neurog3 ${ }^{\text {TA.pUD }}$ and Sox $9^{+}$Neurog3 ${ }^{\text {TA.pLO }}$ populations together-collectively referred to here as the Sox $9^{+}$Neurog3 ${ }^{\text {TA.LO }}$ population (Supplemental Table S1)_represent a mitotic endocrine-
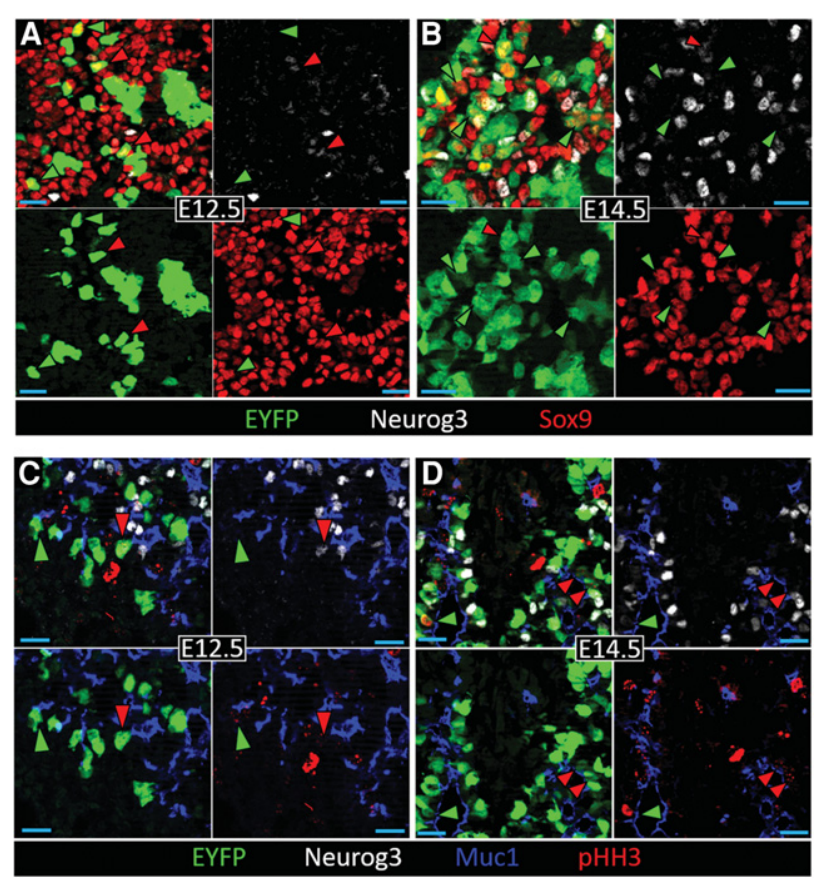

Figure 2. Neurog $3^{\mathrm{TA}}$ Sox $9^{+}$cells, comprising Neurog $3^{\mathrm{TA}} \cdot \mathrm{pLO}$ and Neurog $3^{\text {TA.pUD }}$ cells, represent a mitotic Neurog $3^{\text {TA.LO }}$ endocrine-biased progenitor pool. E12.5 $(A, C)$ and E14.5 $(B, D)$ pancreatic epithelium showing EYFP, Neurog3, and Sox9 or EYFP, Neurog3, Muc1, and pHH3. $(A, B)$ Red and green arrowheads indicate Sox9 $9^{+}$EYFP $^{+}$Neurog3 ${ }^{\text {TA.pLO }}$ cells and Sox $9^{+}$EYFP $^{+}$Neurog3 $3^{\text {TA.pUD }}$ cells, respectively. $(C, D)$ Red and green arrowheads indicate $\mathrm{pHH}^{+} \mathrm{Muc1}^{+}$Neurog3 ${ }^{\mathrm{TA}}$.pLO and $\mathrm{pHH} 3^{+} \mathrm{Muc1}^{+} \mathrm{Neu-}$ rog $3^{\text {TA.pUD }}$ cells, respectively. biased progenitor pool. The evidence from $\mathrm{pHH} 3^{+}$quantification is strengthened below in real-time tracing of this population with a new Neurog3 reporter. In addition, comparing Sox $9^{+}$Neurog $3^{\text {TA.LO }}$ progenitor numbers relative to Sox $9^{-}$lineage-labeled Neurog3 ${ }^{\mathrm{pHI}}$ (referred to here as Neurog3 ${ }^{\text {TA.HI }}$ ) endocrine-committed cells revealed a shift toward Neurog3 ${ }^{\text {TA.HI }}$ cells at E14.5 versus E12.5 (Supplemental Fig. S4), the same pattern as seen in Figure 1D. In sum, we propose that tracking Neurog3 transcriptional activity marks the true intraepithelial Neurog $3^{\mathrm{LO}}$ mitotic progenitor state that is responsible for deriving endocrine-committed Neurog $3^{\mathrm{HI}}$ cells.

\section{Tracking mitotic Neurog3 ${ }^{\text {TA.LO }}$ progenitors via a novel Neurog3 BAC transgenic reporter}

Although the indelible marking of Neurog3-expressing cells by the Neurog $3^{\text {BAC.Cre }}, R 26 R^{\text {EYFP }}$ system is useful for lineage tracing studies, the cumulative nature of this labeling system potentially loses critical aspects of dynamic gene expression. We therefore generated a new, sensitive Neurog3-driven $\mathrm{H}_{2} \mathrm{~B}^{\text {mCherry }}$-peptide-2A-GFP ${ }^{\mathrm{GPI}}$ $\mathrm{BAC}$ transgenic reporter mouse (Neurog ${ }^{\mathrm{RG} 1}$ ) that uses a peptide 2A breaker for simultaneous marking of the chromatin (histone $\mathrm{H} 2 \mathrm{~B}^{\mathrm{mCherry}}$ fusion [ "R"]) and plasma membrane (glycosylphosphatidylinositol [GPI]-EGFP fusion ["G"]) of Neurog3-expressing cells (Supplemental Fig. S5A). The design of the Neurog ${ }^{\text {RG1 }}$ reporter as a BAC transgenic followed several criteria outlined in the Supplemental Material and avoided any negative impact on progenitor behavior caused by engineering the native Neurog3 locus (Wang et al. 2010). Examination of gross tissue morphology and scoring cell type proportional representations-especially focusing on nascent and hormone-expressing endocrine cells over various phases of pancreatogenesis-revealed no abnormal phenotype in Neurog3 ${ }^{\text {RG1 }}$ reporter mice (Supplemental Fig. S5B-E). Glucose levels of ad libitum fed Neurog3 ${ }^{\mathrm{RG} 1}$ mice were similar to wild-type littermates (Supplemental Table S2). The numbers of Sox $9^{+}$Neurog $3^{\mathrm{pLO}}$ cells and Sox $9^{-}$ Neurog $3^{\mathrm{pHI}}$ cells at E12.5 and E14.5 were also unchanged in Neurog $3^{\mathrm{RG} 1}$ transgenic mice relative to wild type (Supplemental Table S2). Rigorous quantification revealed that the majority of Neurog $3^{\text {Protein+ }}$ cells (low and high Neurog $3^{+}$cells together) coexpressed the Neurog $3^{\text {RG1 }}$ reporter $(60 \% \pm 3.2 \%$ at E12.5 and $70 \% \pm 7.7 \%$ at E14.5) (Supplemental Table S2). Although all Neurog $3^{\text {RG1+ }}$ Neurog $3^{\mathrm{pHI}}$ cells showed both mCherry and EGFP signals, the more rapid maturation time of mCherry ( 15 $\mathrm{min}$ ) (Shaner et al. 2005) caused a portion of intraepithelial Neurog $3^{\mathrm{RG} 1+}$ Neurog$^{\mathrm{pLO}}$ cells to be only mCherry ${ }^{+}$(Supplemental Fig. S5B-D); we presume that these cells would later have become red/green dual-fluorescent. Neurog3 expression normally decreases following hormone expression, but the $\mathrm{H}_{2} \mathrm{~B}^{\text {mCherry }}$ longevity and, to a much lesser extent, GFP ${ }^{\mathrm{GPI}}$ caused red/green labeling in some hormone-expressing endocrine cells and an enduring red signal in cells contained within islet clusters (Supplemental Fig. S5D,E). Similar to earlier findings (Fig. 2), at both E12.5 and E14.5, Neurog3 ${ }^{\mathrm{RG} 1}$ transcription reporting 
marked Sox $9^{+}$Neurog $3^{\mathrm{pUD}}$ as well as Neurog $3^{\mathrm{pLO}}$ cells (Figs. 3A,B, 6A), with a significant number of the overall Neurog $3^{\text {TA.LO }}$ population being pHH3 ${ }^{+}$(Fig. 3C,D). Additionally, the number of Sox $9^{+}$Neurog $3^{\text {RG1+ }}{ }^{\text {Neurog }} 3^{\text {TA.LO }}$ cells relative to Sox9- Neurog $3^{\text {RG1+ }}{ }^{-}$Neurog3 ${ }^{\text {TA.HI }}$ cells showed a shift in favor of endocrine commitment at E14.5 versus E12.5 (Fig. 3E), similar to the Neurog $3^{\mathrm{Cre}}$-based lineage tracing result (Supplemental Fig. S4). The higher proportion of $\mathrm{Sox}^{+}$Neurog3 $^{\text {TA.LO }}$ cells relative to Sox9 ${ }^{-}$ Neurog3 $3^{\text {TA.HI }}$ cells (Supplemental Fig. S4 vs. Fig. 3E) likely represents the cumulative labeling over a substantial time period within which Cre activates the binary $R 26 R$ report-
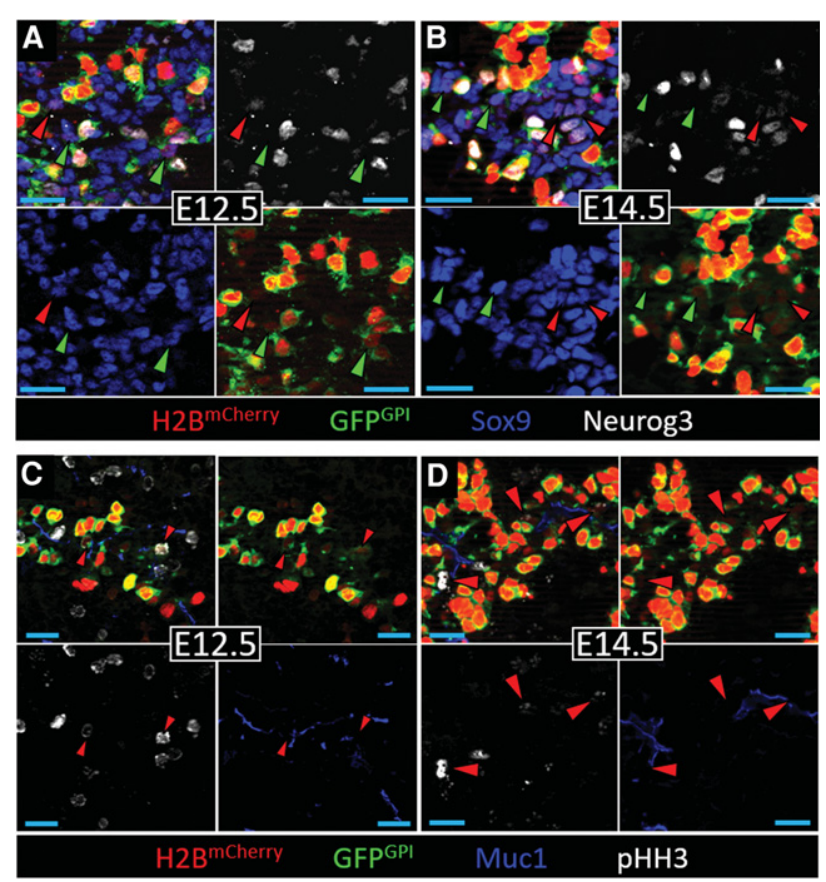

E

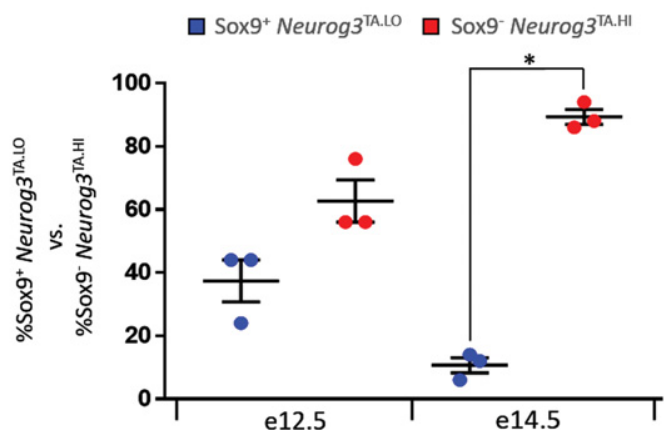

Figure 3. The Neurog ${ }^{\mathrm{RG} 1}$ transgenic reporter marks the mitotic Neurog3 ${ }^{\text {TA.LO }}$ progenitor population. E12.5 $(A, C)$ and E14.5 $(B, D)$ pancreatic epithelium showing $\mathrm{H}_{2} \mathrm{~B}^{\text {mCherry }}$, GFP ${ }^{\mathrm{GPI}}$, Sox9, and Neurog3 or $\mathrm{H}_{2} \mathrm{~B}^{\mathrm{mCherry}}, \mathrm{GFP}^{\mathrm{GPI}}, \mathrm{Muc1}$, and $\mathrm{pHH} 3$. $(A, B)$ Red and green arrowheads indicate $\operatorname{Sox}^{+}$Neurog3 ${ }^{\text {TA.pLO }}$ and Sox $9^{+}$Neurog3 $3^{\text {TA.pUD }}$ cells (seen by low $\mathrm{H}_{2} \mathrm{~B}^{\text {mCherry }}$ signal from Neurog $\left.3^{\mathrm{RG} 1}\right)$, respectively. $(C, D)$ Red arrowheads indicate $\mathrm{Muc1}^{+}$Neurog $3^{\mathrm{RG} 1+} \mathrm{pHH}^{+}$Neurog3 ${ }^{\text {TA.LO }}$ cells. $(E)$ The percentage of Neurog3 ${ }^{\text {RG1+ }}$ Sox $9^{+}$Neurog $3^{\text {TA.LO }}$ versus Neurog3 $3^{\text {RG1+ }}$ Sox $9^{-}$Neurog3 ${ }^{\text {TA.HI }}$ cells at E12.5 $(n=585 ; N=3)$ and E14.5 (n $=6334 ; N=3)$. Data are mean \pm SEM. $\left({ }^{*}\right) P=1 \times 10^{5}$. er, whereas the "snapshot" analysis via the Neurog3 ${ }^{\text {RG1 }}$ reporter might detect only a subset in which the fluorescence is detectable at the time of analysis. These data validate the Neurog $3^{\mathrm{RG} 1}$ mouse line as a passive reporter that correctly marks the various states of Neurog3 expression in the intraepithelial and delaminated stages of endocrine ontogeny.

Comparison of Neurog $3^{\text {TA.LO }}$ progenitors with endocrine-committed cells captured via Neurog $3^{R G 1}$ reporting

The Neurog $3^{\text {RG1 }}$ reporter was immediately valuable in revealing, at relatively high resolution, not only the behavior of intraepithelial Neurog3 ${ }^{\text {TA.LO }}$ progenitors by timelapse imaging but also the derivation and delamination of endocrine-committed Neurog3 ${ }^{\text {TA.HI }}$ cells. During an initial analysis, we observed a previously unknown feature of delaminating Neurog3 ${ }^{\text {TA.HI }}$ cells (Supplemental Fig. S6A). Specifically, Neurog $3^{\text {TA.HI }}$ cells entering delamination showed an early apical membrane narrowing, but later, when most of the cell body had become displaced from the main epithelial layer, there was a pervasive narrow tether-like connection to an almost punctate apical contact at the lumen (Supplemental Fig. S6). Time-lapse imaging of E14.5 Neurog $3^{\text {RG1+ }}$ explants showed that the process of delamination, from initial apical narrowing of a Neurog $3^{\text {TA.HI }}$ cell to its epithelial detachment, lasted upward of $16 \mathrm{~h}$, with a majority of this period spent in the tethered state (Supplemental Fig. S6B,D; Supplemental Movie 1). Quantification of delaminating Neurog $3^{\mathrm{RG} 1-\mathrm{HI}}$ cells showed that $100 \%$ involved apically connected lumenal tethers (CVE Wright, ME Bechard, and E Bankaitis, in prep.; data not shown). The prolonged lumenal contact during delamination of Neurog $3^{\text {TA.HI }}$ cells means that both they and Sox $9^{+} \mathrm{Neu-}$ rog3 ${ }^{\text {TA.LO }}$ cells were $\mathrm{Muc1}^{+}$(Supplemental Fig. S6C). Combining Muc1 positivity with Neurog $3^{\text {RG1 }}$ reporter expression thus provided an opportunity to flow-sort and separate intraepithelial progenitors $\left(\mathrm{Mucl}^{+} \mathrm{Neu}-\right.$ rog $3^{\text {RG1-LO }}$ (Supplemental Table S1) and actively delaminating cells (Muc1 ${ }^{+}$Neurog ${ }^{\text {RG1-HI}}{ }^{\text {) (Supplemental Table }}$ S1) to characterize their progenitor-precursor relationship. To accomplish this, Neurog $3^{\mathrm{RG} 1+}$ E12.5 and E14.5 explants were flow-sorted first for Mucl positivity and then according to low or high Neurog $3^{\text {RG1 }}$ fluorescence (Supplemental Fig. S7A). Analysis via quantitative RTPCR (qRT-PCR) showed that sorted Neurog $3^{\text {RG1-HI }}$ cells from E12.5 and E14.5 pancreata, in addition to negligible Sox 9 expression, were highly enriched for Neurog3, glucagon, insulin (but in E14.5 samples only), Pax6, and NeuroD1 (Supplemental Figs. S7B, S8). Neurog3 ${ }^{\text {RG1-HI }}$ cells were highly enriched for Cdkn1a (Supplemental Fig. S8), which promotes cell cycle exit and is a known target of Neurog3 (Miyatsuka et al. 2011). Recently, Hnf1b was shown to be a critical positive regulator of Neurog3 expression (De Vas et al. 2015). Our analysis, however, shows higher Hnf1b expression in Neurog3 ${ }^{\text {TA.HI }}$ cells versus Neurog3 ${ }^{\text {TA.LO }}$ cells (Supplemental Fig. S8), which could indicate a role in activating Neurog3 expression 
during the early Neurog3 ${ }^{\text {TA.LO }}{ }^{\text {-to-Neurog3 }} 3^{\text {TA.HI }}$ transition. These data are consistent with actively delaminating Muc1 $^{+}$Neurog3 ${ }^{\text {RG1-HI }}$ cells being post-mitotic, undergoing endocrine commitment toward the hormone-expressing condition. In contrast, sorted Neurog3 ${ }^{\text {RG1-LO }}$ cells had significantly lower Neurog3 expression and negligible glucagon, Pax6, or NeuroD1 expression, with significant enrichment in Sox9, Hes1, and Hnf6 (Supplemental Figs. S7B, S8). Consistent with their mitotic nature, cyclin-dependent kinase 6 (Cdk6; a positive cell cycle regulator) was enriched in Neurog $3^{\text {RG1-LO }}$ cells (Supplemental Fig. S8). Because Sox9, Hes1, and Hnf6 are accepted progenitor-state markers within the bipotent population of the trunk epithelium, these data fit with the Sox $9^{+}$Neurog $3^{\text {TA.LO }}$ population being at or close to a progenitor state.

\section{Real-time analysis of mitotic Neurog $3^{\text {TA.LO }}$ cells}

To investigate further our proposal that $\mathrm{Sox}^{+} \mathrm{Neu-}$ rog $3^{\text {TA.LO }}$ cells give rise to both Neurog $3^{\text {TA.LO }}$ progenitors and endocrine-committing post-mitotic Neurog3 ${ }^{\text {TA.HI }}$ precursors, E12.5 Neurog3 ${ }^{\mathrm{RG} 1}$ explants were subjected to time-lapse imaging focusing on intraepithelial low-level fluorescing cells. Dividing Neurog3 ${ }^{\text {TA.LO }}$ cells and their progeny were followed for $\sim 12-24 \mathrm{~h}$ (Supplemental Table S3); imaging was restricted to $\sim 24 \mathrm{~h}$ because the end of this period started to show presumably phototoxic effects on tissue growth and cell survival, a known consequence of confocal laser exposure (Stephens and Allan 2003). The size of the pancreatic explants ( 50-100 $\mu \mathrm{m}$ thick) and substantial light scattering in deep-tissue imaging served to limit our analysis to $\sim 30 \mu \mathrm{m}$ closest to the microscope objective (Supplemental Table S3). Within this depth, we observed an average of three intraepithelial Neurog3 ${ }^{\mathrm{RG1-LO}}$ mitotic events per explant-a total of 15 over five real-time imaging runs (Supplemental Table S3). Although an apical marker was absent during our real-time analysis, a Neurog $3^{\text {TA.LO }}$ cell was assigned as intraepithelial by the extent of its apical surface, the lack of the narrow tether seen on delaminating Neurog $3^{\text {RG1-HI }}$ cells, and nuclear proximity to the lumen (Fig. 4A,B). Several factors likely contributed to observing what is probably a subset of the true number of Sox $9^{+}$Neurog $3^{\text {TA.LO }}$ cell divisions occurring within the pancreatic epithelium in vivo. First, the relatively high number of Neurog $3^{\mathrm{RG} 1+}$ cells and high fluorescence in Neurog3 ${ }^{\mathrm{RG} 1-\mathrm{HI}}$ cells in the E12.5E13.5 period meant that divisions were clearly discernible only in regions less densely populated by Neurog3 ${ }^{\mathrm{RG} 1+}$ cells. Second, the pancreatic epithelium is approximately fourfold less mitotically active under ex vivo culture conditions (Puri and Hebrok 2007; Kim et al. 2015). To determine whether the observed division events produced Neurog $3^{\text {TA.LO }}$ cells or Neurog $3^{\text {TA.HI }}$ cells, the level of $\mathrm{H}_{2} \mathrm{~B}^{\text {mCherry }}$ fluorescence intensity in the nucleus of an intraepithelial Neurog $3^{\mathrm{RG} \text {-LO }}$ cell was quantified first at the initial appearance of mitotic chromosomes (Supplemental Table S3). Although details of $\mathrm{H}_{2} \mathrm{~B}^{\text {mCherry }}$ fluorescence are reported for this analysis, the GFP
A

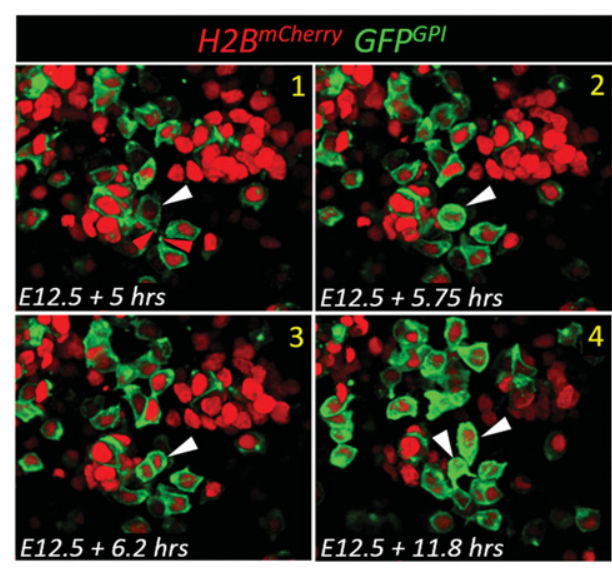

B

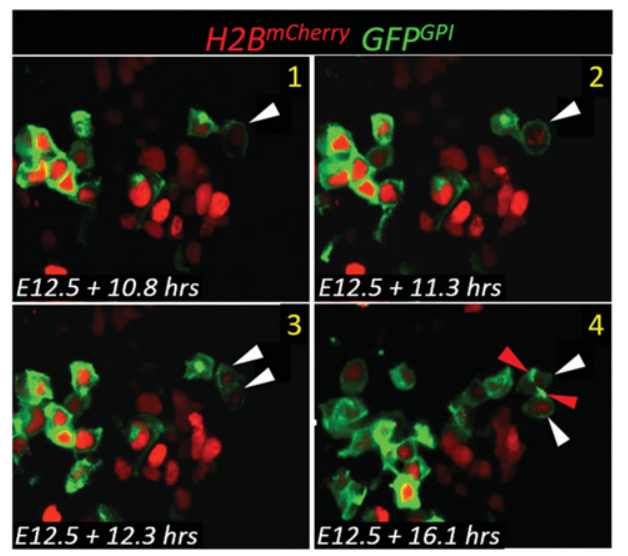

Figure 4. Division of Neurog3 $3^{\text {TA.LO }}$ progenitors in real time. Division of a Neurog3 ${ }^{\text {TA.LO }}$ cell into two delaminating Neurog $3^{\text {TA.HI }}$ cells $(A)$ or two Neurog $3^{\text {TA.LO }}$ progenitors $(B)$. Images are screen captures from Fig. 4B; Supplemental Movie 2. White and red arrowheads indicate a Neurog3 ${ }^{\text {TA.LO }}$ division event and apical attachment, respectively.

fluorescence intensity provided matching information. Next, the fluorescence intensity of Neurog $3^{\text {RG1 }}$-expressing daughters was quantified $\sim 3-6 \mathrm{~h}$ after division, with fluorescence intensity quantification beyond $6 \mathrm{~h}$ being dependent on how long the daughter cells were tracked before video termination or whether they moved out of view (Supplemental Table S3). Neurog3 ${ }^{\text {RG1+ }}$ daughter cells were determined to have initiated delamination if they increased the Neurog $3^{\mathrm{RG} 1}$ fluorescence intensity or showed basal-ward displacement of their nucleus/cell body or other signs of delamination, such as basally directed membrane-protrusive behavior, formation of the lumenal tether, and greatly narrowed apical contact. By these parameters, two division categories were observed. In the first, a Neurog $3^{\text {RG1-LO }}$ cell produced two daughters, each increasing Neurog $3^{\mathrm{RG} 1}$ reporter fluorescence intensity by an average of $\sim 2.5$-fold and simultaneously exhibiting signs of delamination (Fig. 4A; Supplemental Table S3; Supplemental Movie 2). This division was a symmetric division terminal differentiation event in which both 
daughters moved toward endocrine commitment, with the transition from Neurog3 ${ }^{\text {TA.LO }}$ to Neurog3 ${ }^{\text {TA.HI }}$ (as marked by the Neurog $3^{\text {RG1 }}$ reporter) taking $\sim 3-6$ h. In the second type of division, a Neurog $3^{\text {RG1-LO }}$ cell produced two daughters that did not increase Neurog3 ${ }^{\text {RG1 }}$ reporter fluorescence intensity, exhibited no filopodial exploratory behavior at the basal surface or other signs of delamination, and remained in an intraepithelial location (Fig. 4B; Supplemental Table S3; Fig. 4B; Supplemental Movie 3). This type of division was classified as symmetric division progenitor maintenance: a Neurog $3^{\text {TA.LO }}$ progenitor producing daughters that both remained in the Neurog $3^{\text {TA.LO }}$ progenitor state. Intraepithelial Sox $9^{+}$Neurog $3^{\text {TA.LO }}$ progenitors are thus able to undergo divisions that either produce more progenitors or give rise to more differentiated progeny. Of the observable mitotic events scorable during these movies, all occurred in Neurog3 ${ }^{\text {TA.LO }}$ (Neurog3 ${ }^{\text {RG-LO) }}$ cells; no Neurog3 ${ }^{\text {RG-HI }}$ divisions were observed. There was an overall 53\%:47\% split between progenitor maintenance and symmetric terminal differentiation divisions, and no asymmetric cell divisions were observed, forming one Neurog $3^{\text {TA.LO }}$ and one Neurog $3^{\text {TA.HI }}$ cell. We consider these data to be in accordance with the incipient building up of the endocrine-biased progenitor pool during the rapid plexus expansion occurring at this stage of pancreas organogenesis.

Although not directly observed in our time-lapse movies (possibly because of the restricted time-lapse period and the slowing of mitotic activity ex vivo), we hypothesized that mitotic Neurog $3^{\text {TA.LO }}$ progenitors in vivo can undertake multiple rounds of division to produce, over time, considerable numbers of Neurog3 ${ }^{\text {TA.LO }}$ progenitors or Neurog3 ${ }^{\text {TA.HI }}$ cells. Sequential dual-thymidine analog labeling is one way of tracking cells that divide more than once (Teta et al. 2007; Bradford and Clarke 2011). To determine the relative rate at which multiple rounds of progenitor maintenance versus terminal differentiation occurred, we quantified the relative proportion of EdU/ BrdU double-labeled Neurog $3^{\mathrm{pLO}}$ cells versus EdU/BrdU double-labeled Neurog $3^{\mathrm{pHI}}$ cells. To accomplish this, E12.5 or E14.5 pancreatic epithelium was labeled sequentially with single EdU and BrdU injections $12 \mathrm{~h}$ apart (Supplemental Fig. S9A). To avoid fluorescence crossover problems when combining mCherry/GFP signals from Neurog3 ${ }^{\text {RG1 }}$ with EdU/BrdU immunofluorescence signals, we used Neurog3 protein to define Neurog3 ${ }^{\text {TA.LO }}$ and Neurog3 ${ }^{\text {TA.HI }}$ cells. Despite the likely underscoring of Neurog3 ${ }^{\text {TA.LO }}$ cells by using this method (for reasons described above), we show that the percentage of $\mathrm{EdU}^{+} \mathrm{BrdU}^{+}$Neurog $3^{\mathrm{pLO}}$ cells is $\sim 3.2$-fold higher than that of $\mathrm{EdU}^{+} \mathrm{BrdU}^{+}$Neurog $3^{\mathrm{pHI}}$ cells at E12.5, compared with equal proportions of the two populations at E14.5 under the same labeling scheme (Supplemental Fig. S9B,D, E). It is possible that the complex plexus-state epithelium in vivo displays spatiotemporally varied behavior of Neurog $3^{\text {TA.LO }}$ progenitors with respect to their frequency of undergoing multiple rounds of division. Our snapshotstyle bulk analysis will therefore tend toward a "homogenizing" nature when scoring across such a largely asynchronous epithelium and underrepresent the variation in mitotic activity of Neurog3 $3^{\text {TA.LO }}$ progenitors as the plexus-state endocrine progenitor niche develops, moves toward a state of peak endocrine yield, and begins final remodeling to the mature ductal tree. Nevertheless, these data fit the notion that, early in the secondary transition, progenitor maintenance-type division of the $\mathrm{Sox}^{+} \mathrm{Neu-}$ rog $3^{\text {TA.LO }}$ pool is favored over symmetric terminal differentiation-type division (Supplemental Fig. S9C). As the pancreatic epithelium progresses through the secondary transition, however, the proportion of terminal differentiation events could increase to accommodate local increases in endocrine cell birth rate.

\section{Limiting Neurog3 expression increases proliferation} and expands the Neurog $3^{\text {TA.LO }}$ progenitor pool

We tested whether deliberately reducing the level of Neurog3 gene activity would increase the proliferative index of Neurog $3^{\text {TA.LO }}$ progenitors and/or cause a concomitant population expansion. We determined the number and mitotic activity of Sox $9^{+}$Neurog3 ${ }^{\text {TA.LO }}$ cells in a decreasing series of gene dosages: wild-type, Neurog3 heterozygous (Neurog $3^{\mathrm{EGFP} /+}$ ), and strong Neurog3 hypomorphic $\left(\right.$ Neurog $3^{\text {EGFP/FL }}$ ) conditions. At E14.5, Neurog3 expression and protein levels in the developing pancreas are known to decrease by $\sim 50 \%$ in the heterozygous condition and $\sim 75 \%$ under the strong hypomorphic condition (Wang et al. 2010) and are known to release Neurog3-expressing cells to nonendocrine lineages in both "reduced dosage" states. In that study, a large increase in Neurog3-expressing epithelial cells occurred in the strong hypomorphic condition compared with the heterozygous condition (Wang et al. 2010). However, the Neurog3 ${ }^{\text {EGFP }}$ reporter that they used was Neurog3-null, preventing comparison with wild-type tissues. Our new Neurog3 ${ }^{\text {RG1 }}$ reporter does not perturb the endogenous locus and thus allows this comparison. We reasoned that the Neurog $3^{\mathrm{RG} 1}$ BAC transgene reporter (see the Supplemental Material) would behave similarly to the endogenous locus EGFP knock-in for estimating dynamic behavior of the Neurog $3^{\text {TA.LO }}$ population. We decided against using Neu$\operatorname{rog} 3^{\text {BAC.Cre }} ; 226 R^{\text {EYFP }}$ lineage tracing in the wild-type condition, which captures the population cumulatively via a dual-gene activation system. In areas of plexus-like epithelium that are rich in endocrine-committing and delaminating Neurog $3^{\mathrm{HI}}$ cells, the visual estimation of the prevalence of the intraepithelial Neurog3 ${ }^{\text {TA.LO }}$ population was similar for Neurog3 ${ }^{\text {RG1 }}$ (Fig. 3A,B) and Neurog3 ${ }^{\text {EGFP/+ }}$ tissue (Fig. 5A,B), which was then validated by quantification across the epithelium (Fig. 6C), supporting the idea that both are effective for probing the population behavior of the Neurog3 ${ }^{\text {TA.LO }}$ pool. In all three genotypes-wild type (containing Neurog $3^{\mathrm{RG} 1+}$ ), heterozygous Neurog $3^{\mathrm{EGFP} /+}$, and strong hypomorphic Neurog3 ${ }^{\mathrm{EGFP} / \mathrm{FL}}$ we could easily observe Neurog3 ${ }^{\text {TA.pLO }}$ (Neurog3 protein-low) and Neurog3 ${ }^{\text {TA.pUD }}$ (Neurog3 protein-undetectable) cells. As for the wild-type tissue, the heterozygous and the strong hypomorph conditions also showed significant numbers of their overall $\mathrm{EGFP}^{+} \mathrm{Sox}^{+} \mathrm{Neu-}$ rog $3^{\text {TA.LO }}$ population as $\mathrm{pHH}^{+}{ }^{+}$(Fig. 5E-H). Quantification 


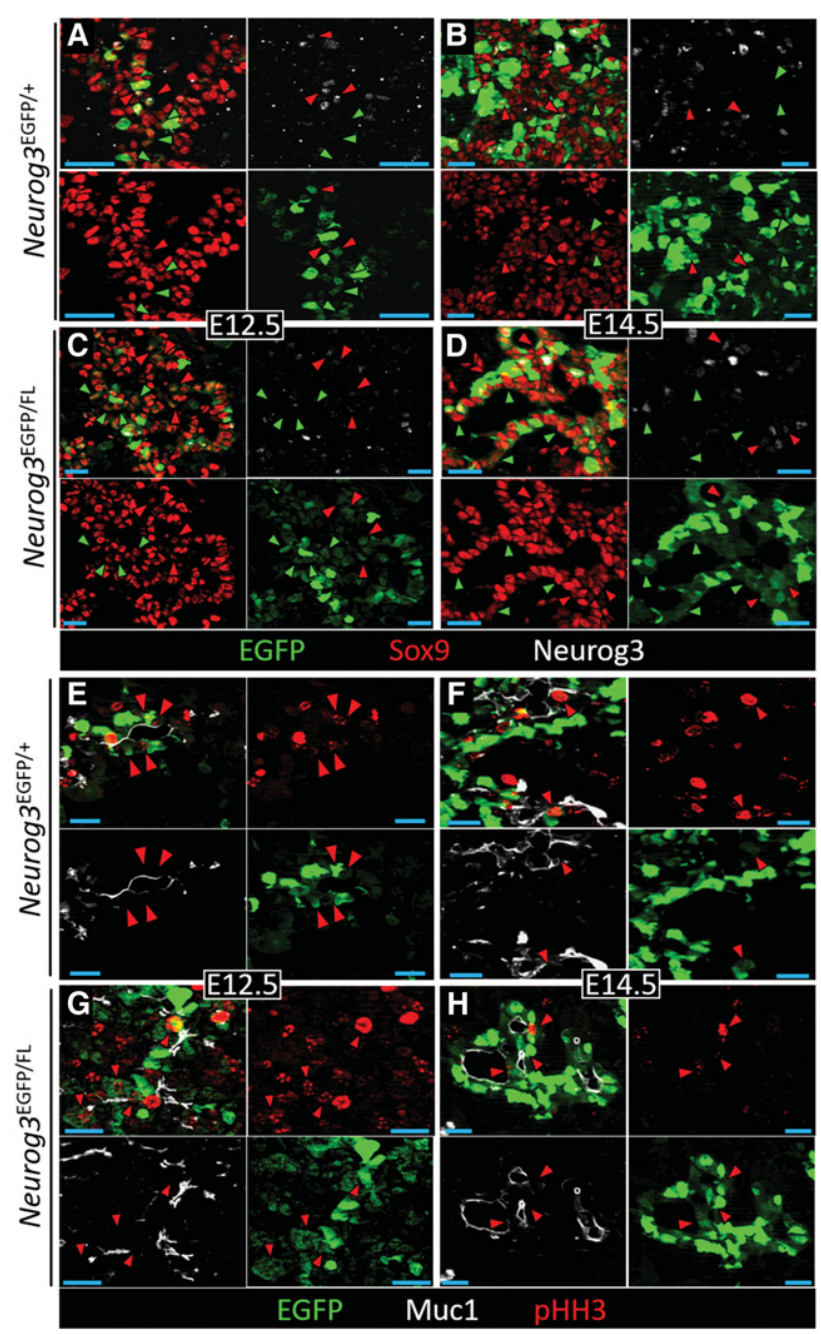

Figure 5. The mitotic Neurog $3^{\text {TA.LO }}$ population is prevalent under Neurog3 heterozygous and strong hypomorphic conditions. E12.5 $(A, C, E, G)$ and E14.5 $(B, D, F, H)$ pancreatic epithelium showing EGFP, Neurog3, and Sox9 or EGFP, Muc1, and pHH3 from Neurog $3^{\text {EGFP/+ }}$ and Neurog3 ${ }^{\text {EGFP/FL }}$ embryos. $(A-D)$ Red and green arrowheads indicate Sox $9^{+}$Neurog $3^{\text {TA.pLO }}$ and Sox $9^{+} \mathrm{Neu-}$ rog $3^{\text {TA.pUD }}$ cells (marked by low EGFP signal from Neurog $3^{\text {EGFP }}$ ), respectively. $(E-H)$ Red arrowheads indicate $\mathrm{Mucl}^{+} \mathrm{EGFP}^{+}$ pHH3 $3^{+}$Neurog3 ${ }^{\text {TA.LO }}$ cells.

showed that, relative to the wild type, the number of Sox $9^{+}$Neurog3 ${ }^{\text {TA.pUD }}$ cells was significantly (and equivalently) greater than Sox $9^{+}$Neurog $3^{\text {TA.pLO }}$ cells under Neurog3 heterozygous and strong hypomorphic conditions at both E12.5 and E14.5 (Figs. 5C,D, 6A). We also observed, at E12.5, a significantly increased Sox $9^{+}$Neurog $3^{\text {TA.LO }}$ progenitor pool (combining protein-low and protein-undetectable cells) relative to the endocrine-committed Neurog3 ${ }^{\text {TA.HI }}$ pool in the heterozygous and strong hypomorphic conditions (cf. Figs. 3E and 6B). As described above (Figs. 1D, 3E), wild-type tissue showed a significantly increased Neurog3 ${ }^{\text {TA.HI }}$ relative to Neurog3 ${ }^{\text {TA.LO }}$ cell number between E12.5 and E14.5. The degree of this temporal shift toward the Neurog3 ${ }^{\text {TA.HI }}$ pool was much re- duced in the heterozygous condition and nearly absent in the strong hypomorph (Figs. 3E vs. 6B). These results suggest that limiting Neurog3 expression-and thus protein levels-increased the number of Sox $9^{+}$Neurog $3^{\text {TA.LO }}$ progenitors undergoing progenitor maintenance divisions relative to terminal differentiation-type divisions.

We next determined whether the increased production of Sox $9^{+}$Neurog3 ${ }^{\text {TA.LO }}$ progenitors under reduced gene dosage affected the overall proportional representation of the intraepithelial Neurog3 ${ }^{\text {TA.LO }}$ progenitor population. Compared with the wild-type (Neurog $3^{\mathrm{RG1+}}$ ) control, the proportion of Sox $9^{+}$Neurog $3^{\text {TA.LO }}$ cells under the heterozygous condition, although unchanged at E12.5, was 2.5fold expanded at E14.5 (Fig. 6C). Under the strong hypomorphic condition, however, this population was increased eightfold at E12.5 and 14-fold at E14.5 (Fig. 6C). These expansions were associated with increased proliferation as marked by $\mathrm{pHH}$. As explained above (Fig. 2), only Neurog3 ${ }^{\mathrm{TA}}$ cells (Neurog3 ${ }^{\mathrm{RG} 1}$-positive cells) with clear $\mathrm{Mucl}^{+}$surfaces were defined as intraepithelial and with DAPI as an aid where appropriate. The mitotic index of Neurog3 $3^{\text {TA.LO }}$ cells in Neurog $3^{\text {EGFP/+ }}$ heterozygous relative to the wild type, although unchanged at E12.5, was increased 1.4-fold at E14.5 (Fig. 7A). In the Neurog3 ${ }^{\text {EGFP/FL }}$ strong hypomorph, however, the index was increased 2.1-fold at E12.5 and 1.7-fold at E14.5 (Fig. 7A). We also scored for any non-cell-autonomous effect of limiting Neurog3 expression on the mitotic index of the surrounding Neurog3-nonexpressing $\left(\mathrm{Mucl}^{+} \mathrm{Neurog}^{-}\right)$epithelium but found no significant change (Fig. 7A). These findings are consistent with a low level of Neurog3 protein having a critical role in the maintenance of an actively cycling, endocrine-biased Neurog $3^{\text {TA.LO }}$ progenitor state (Fig. 7B).

\section{Discussion}

We describe several lines of evidence pointing to a new model for the generation of endocrine-committed Neurog $3{ }^{\mathrm{HI}}$ cells during the pancreatic secondary transition, driven by an intraepithelial endocrine-biased mitotic Neurog3 ${ }^{\text {TA.LO }}$ progenitor population (Fig. 7B). Using Neurog3-driven transcriptional reporters, we show that these progenitors are relatively abundant and have prolonged residency within the plexus-state epithelium of the developing pancreas, undertaking symmetric divisions to maintain the progenitor pool or produce two endocrinecommitting daughters. As a mitotic pool that continually feeds production of endocrine-committed cells by symmetric terminal differentiation division, its behavior is reminiscent of progenitors in other tissues (for example, the intestinal crypt), acting as a transit-amplifying population. We do not propose that the Neurog $3^{\text {TA.LO }}$ progenitor pool is set aside at a very early stage of pancreas development, within the primary transition. However, further investigation (probably requiring lineage tracing tools capable of labeling only Neurog3 low-level expressors) would be needed to distinguish whether the Sox $9^{+} \mathrm{Neu-}$ rog $3^{\text {TA.LO }}$ endocrine-biased progenitor pool continues to 
A

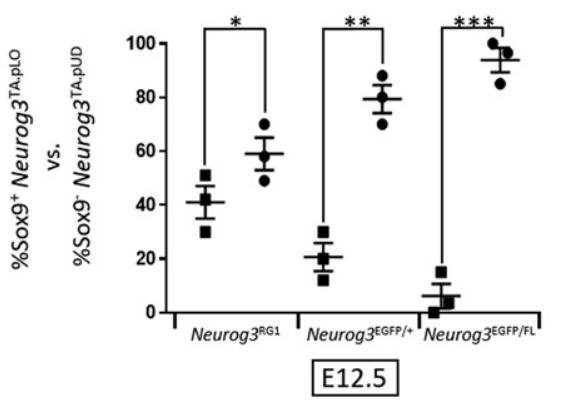

B

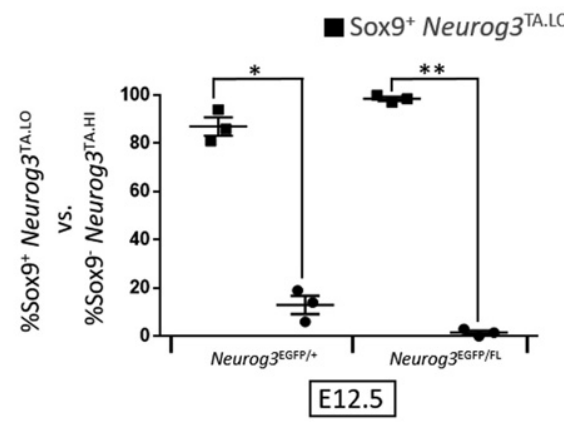

C

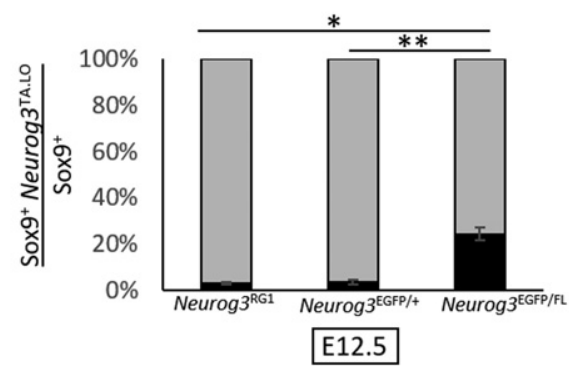

Sox9 ${ }^{+}$Neurog $3^{\text {TA.plo }}$

- Sox9 ${ }^{+}$Neurog3 $3^{\text {TA.pud }}$

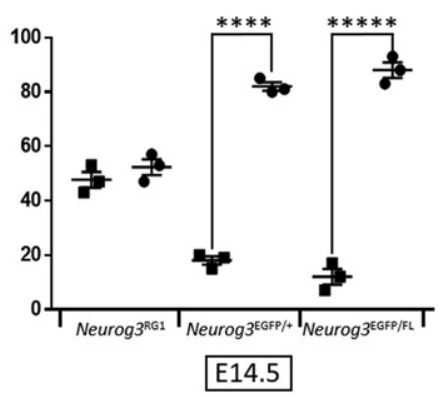

Sox9- Neurog3 $3^{\text {TA.HI }}$
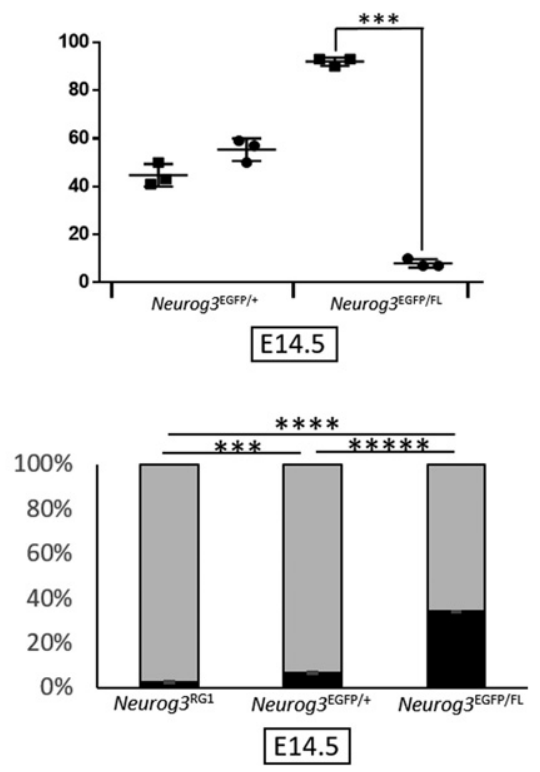

Figure 6. Limiting Neurog3 expression expands the Neurog3 $3^{\text {TA.LO }}$ population at the expense of Neurog $3^{\text {TA.HI }}$ cells. $(A)$ The percentage

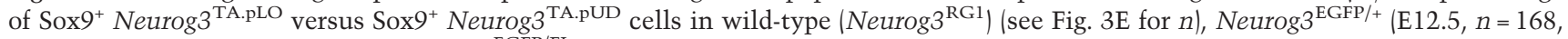
$N=3 ; \mathrm{E} 14.5, n=2425, N=3)$, and Neurog3 ${ }^{\mathrm{EGFP} / \mathrm{FL}}(\mathrm{E} 12.5, n=1237, N=3 ; \mathrm{E} 14.5, n=5818, N=3)$ pancreata. $\left({ }^{*}\right) P=0.0523 ;\left({ }^{* *}\right) P=0.00067$; $\left.\left.{ }^{* * *}\right) P=8.3 \times 10^{-5} ;{ }^{* * * *}\right) P=4 \times 10^{-6} ;\left(^{* * * * *}\right) P=2.5 \times 10^{-5}$. $(B)$ The percentage of Sox $9^{+}$Neurog3 ${ }^{\text {TA.LO }}$ versus Sox $9^{-}$Neurog $3^{\text {TA.HI }}$ cells in Neurog $3^{\mathrm{EGFP} /+}$ and Neurog3 ${ }^{\mathrm{EGFP} / \mathrm{FL}}$ (see $A$ for $n$ ) pancreata. $\left.\left.\left({ }^{*}\right) P=8 \times 10^{-5} ;{ }^{* *}\right) P=8 \times 10^{-8} ;{ }^{* * *}\right) P=2.4 \times 10^{-7}$. (C) The percentage of Sox $9^{+}$cells that are Neurog3 ${ }^{\mathrm{TA} . \mathrm{LO}}$ in wild-type (Neurog $\left.3^{\mathrm{RG} 1}\right)(\mathrm{E} 12.5, n=4497, N=3 ; \mathrm{E} 14.5, n=11930, N=4)$, Neurog3 $^{\mathrm{EGFP} /+}(\mathrm{E} 12.5, n=$ 4718; E14.5, $n=15903)$, and Neurog3 ${ }^{\mathrm{EGFP} / \mathrm{FL}}(\mathrm{E} 12.5, n=4656 ; \mathrm{E} 14.5, n=15671)$ pancreata. $\left({ }^{*}\right) P=0.00015 ;\left(^{* *}\right) P=0.0009 ;\left(^{* * *}\right) P=0.0022$; ${ }^{(* * *)} P=2 \times 10^{-7} ;(* * * *) P=1 \times 10^{-6}$. Data are mean \pm SEM.

be established throughout the secondary transition by derivation from a parental bipotent (duct/endocrine) trunk epithelium population or is essentially completely allocated within a short period near the inception of the secondary transition. If the latter result were to be found, it would support one interpretation of a previous lineage tracing study (Gu et al. 2002) showing that a large-scale separation of the duct and endocrine progenitors occurs in the run-up to the secondary transition as the plexusstate trunk epithelium becomes organized. The intraepithelial Neurog3 ${ }^{\text {TA.LO }}$ pool is likely maximally estimated through the use of Neurog3 ${ }^{\text {BAC.Cre }} ; R 26 R^{\text {EYFP }}$ lineage labeling, in which we determined that, at E14.5, about one-quarter of the whole Sox $9^{+}$epithelium was in the Neurog $3^{\text {TA.LO }}$ state. These cells were often clustered to- gether, not scattered homogeneously, and scoring specifically plexus-state epithelial cells revealed up to half of them in this state. Our snapshot-type longitudinal analysis leaves open the possibility that any individual area of the plexus might have juvenile, mature, and aged phases in which the proportional representation of Neurog3 ${ }^{\text {TA.LO }}$ cells varies (according to developmental inputs), with all regions at some time passing through a "peak occupancy" period. Regarding our finding that reducing Neurog3 expression led to expansion of the Sox $9^{+}$Neurog3 $3^{\text {TA.LO }}$ progenitor population and significantly increased its mitotic index, these effects on the Neurog3 ${ }^{\text {TA.LO }}$ pool may also be underestimated by our scoring large regions of epithelium without being able to refer to spatial variations in the relative maturity of individual epithelial regions. 
A

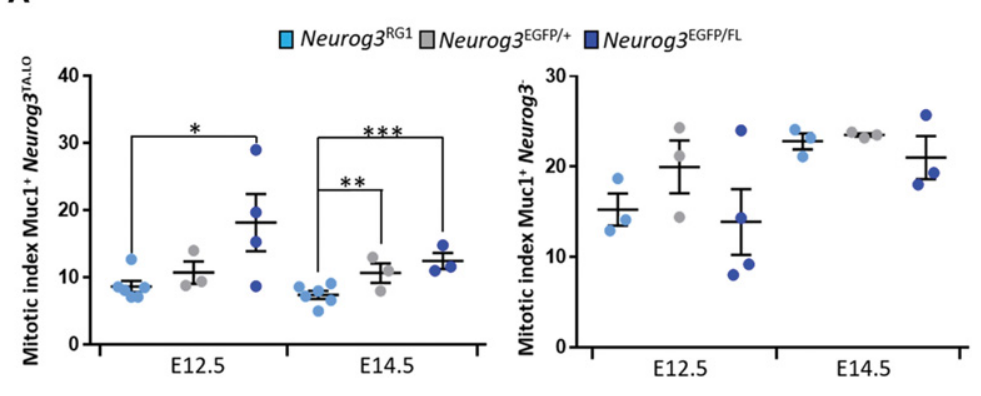

B

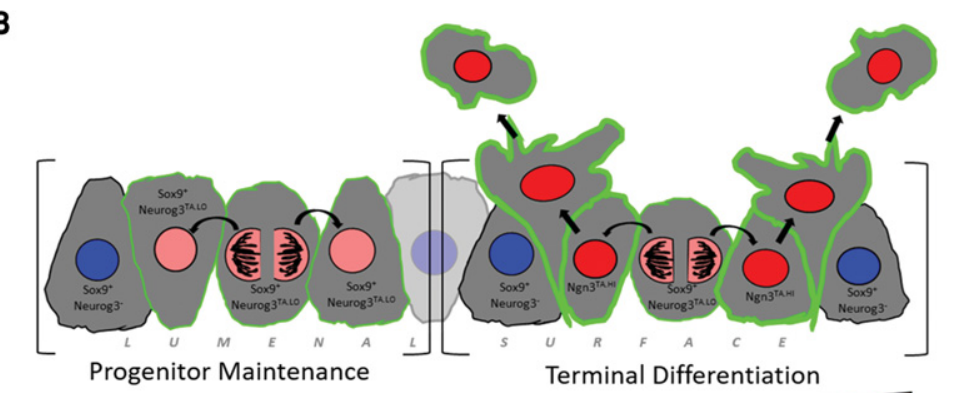

Neurog3 Protein Levels
Figure 7. Reducing Neurog3 expression increases the mitotic index of Sox $9^{+}$Neurog $3^{\text {TA.LO }}$ progenitors. $(A$, left) The mitotic index (percentage $\mathrm{pHH}^{+}$) of $\mathrm{Muc1}^{+}$ Neurog $3^{\text {TA.LO }}$ cells under wild-type (Neurog3 ${ }^{\text {RG1 }}$ ) (E12.5, $n=569, N=6 ;$ E14.5, $n=2401, N=6)$, Neurog3 ${ }^{\mathrm{EGFP} /+}(\mathrm{E} 12.5, n=362, N=3 ; \mathrm{E} 14.5, n=904, N=3)$, and Neurog $3^{\mathrm{EGFP} / \mathrm{FL}}(\mathrm{E} 12.5, n=783, N=4 ; \mathrm{E} 14.5, n=$ 3905, N=3) conditions. (Right) The mitotic index of Muc1 $^{+}$Neurog3 ${ }^{-}$cells in wild-type (Neurog3 ${ }^{\text {RG1 }}$ ) (E12.5, $n=1200$ cells, $N=3$; E14.5, $n=7490, N=3)$, Neu$\operatorname{rog} 3^{\mathrm{EGFP} /+}(\mathrm{E} 12.5, n=1003, N=3 ; \mathrm{E} 14.5, n=2737, N=3)$ and Neurog3 ${ }^{\mathrm{EGFP} / \mathrm{FL}}(\mathrm{E} 12.5, n=2226, N=4 ; \mathrm{E} 14.5, n=$ 5217, $N=3$ ) pancreata. Data are mean \pm SEM. (*) $P=$ 0.0138 ; $\left.{ }^{* *}\right) P=0.0207$; $\left.{ }^{* * *}\right) P=0.0018$. (B) Model depicting the behavior of Neurog $3^{\text {TA.LO }}$ cells in the pancreatic epithelium.
Our findings fit better with aspects of Neurog3 expression data reported in previous literature that were not previously highlighted, providing a new level of clarity regarding the role of Neurog3 in directing endocrine cell production during the secondary transition. Below, we speculate that our findings may indicate an interesting parallel with the role of the Neurog3 sister protein Neurog2 in neuronal development. Taking into account that the final endocrine cell fate choice is thought to have occurred in Neurog3-expressing cells (Desgraz and Herrera 2009), future experiments should investigate whether lineage specification/commitment might occur in the Neurog $3^{\text {TA.LO }}$ endocrine-biased progenitor state. Such information could advance efforts to manipulate human pluripotent stem cell-derived endocrine progenitors into producing specific types and quantities of functional endocrine cells.

Low Neurog3 expression defines an endocrine-biased mitotic progenitor state in Sox $9^{+}$cells

Our conclusion that Sox $9^{+}$Neurog3 $3^{\text {TA.LO }}$ progenitors represent an actively cycling, discrete, endocrine-biased progenitor population is consistent with previous in situ hybridizations using probe cocktails marking early steps of endocrine ontogeny to show broad mRNA distributions across most of the E14.5 epithelium (Zhou et al. 2007). These data corroborate the notion that many intraepithelial cells have entered the endocrine lineage program, maybe even in a relatively concerted manner beyond activating only Neurog3 expression (Zhou et al. 2007). Neu- rog3 $3^{\text {BAC.Cre }}$-based lineage tracing in wild-type tissue shows that almost all Neurog $3^{+}$cells move eventually into the endocrine lineage, with a small percentage adopting nonendocrine acinar or duct lineages (Schonhoff et al. 2004). Furthermore, reducing Neurog3 expression via heterozygous/hypomorphic alleles significantly decreases the degree of endocrine biasing, allowing increased drift to nonendocrine lineages (Wang et al. 2010). Fitting our results with these studies prompts the conclusion that normal Neurog3 ${ }^{\text {TA.LO }}$ cells represent an endocrine-biased progenitor state that retains a small degree of multipotency. It will be interesting to determine, perhaps via single-cell RNA sequencing analysis, whether a lowlevel "sampling" type of expression activates, in Neurog3 ${ }^{\text {TA.LO }}$ endocrine-biased progenitors, genes lying downstream in the endocrine lineage specification-commitment-differentiation program, such as Insm1A, Pax4, Nkx2.2, and Arx. The notion that Neurog3 ${ }^{\text {TA.LO }}$ progenitors are noncommitted but nonetheless access endocrine pathway programs targeted by Neurog3 is analogous to the concept that, during hematopoiesis, progenitor cells are selectively primed toward multiple lineage programs (Nimmo et al. 2015).

Our confirmation that almost all Neurog3 $3^{\text {TA.HI }}$ cells are post-mitotic fits with previous studies demonstrating that high Neurog3 protein levels instruct cell cycle exit and transition to a unipotent precursor state producing an individual endocrine cell type (Desgraz and Herrera 2009). We propose that those previous studies focused on endocrine-committed Neurog $3^{\mathrm{HI}}$ cells, not low-level epithelial Neurog $3^{\text {TA.LO }}$ cells, because certain lineage tracer 
Neurog3 $3^{\text {Cre }}$ driver/reporter pairings (for example, the Neurog3 ${ }^{\mathrm{Cre}}{ }_{;} \mathrm{Z} / \mathrm{AP}$ reporter system) (Gu et al. 2002) require a higher level of Neurog3 ${ }^{\mathrm{Cre}}$ expression that is reached only during endocrine commitment, thus missing the low-level-expressing intraepithelial cells. It is plausible that the increased ability of the Neurog $3^{\text {BAC.Cre }}$ (Schonhoff et al. 2004) to switch on R26R-based lineage reporters relates to cis-regulatory regions present in the BAC transgene that augment the known enhancer promoter elements of Neurog3 (Lee et al. 2001; Ejarque et al. 2013). Likely, our selection of the same Neurog3 BAC used by Schonhoff et al. (2004) in our Neurog $3^{\mathrm{RG1}}$ reporter facilitated study of the Neurog $3^{\text {TA.LO }}$ progenitor population.

Low Neurog3 protein levels promote a mitotic endocrinebiased Neurog $3^{\text {TA.LO }}$ progenitor state

Our new model is that abundant Neurog $3^{\text {TA.LO }}$ progenitors are maintained in a mitotic endocrine-biased state within plexus-state epithelial regions until they enter plexus-to-duct resolution (Bankaitis et al. 2015) using, in the interim, symmetric cell division to both maintain the endocrine-biased progenitor state and derive endocrine-committing Neurog $3^{\text {TA.HI }}$ cells. There was an apparent absence of asymmetric divisions that yield one Neurog3 ${ }^{\text {TA.LO }}$ and one Neurog3 ${ }^{\text {TA.HI }}$ daughter. Recently, Kim et al. (2015) published a real-time analysis of mitotic $P d \times 1^{\mathrm{GFP}}$ - and Neurog $3^{\mathrm{RFP}}$-expressing cells. They reported division events for $P d x 1$-expressing cells that were symmetric (for both progenitor renewal and terminal differentiation) and asymmetric (producing one $\mathrm{Pdx}^{+} \mathrm{Sox} 9^{+}$ daughter and one Neurog $3^{+}$daughter). Similar to our observations, there was no asymmetric division for mitotic Neurog3-expressing cells. Furthermore, our results suggest that, at E12.5, perhaps connected to the rapid building and expansion of the incipient plexus-state epithelium occurring at this stage, the proportion of Neurog3 ${ }^{\text {TA.LO }}$ cells undergoing progenitor maintenance divisions relative to terminal differentiation events is higher than at E14.5. Limiting Neurog3 expression in the strong hypomorphic condition increased the mitotic index of $\mathrm{Sox}^{+} \mathrm{Neu-}$ rog $3^{\text {TA.LO }}$ progenitors and concurrently expanded their proportional representation within the epithelium at the expense of endocrine commitment (Fig. 6B,C), perhaps reflecting a shift in favor of progenitor-maintaining divisions. Recently, we reported that ablating Neurog3 expression decreased the proliferation rate of the entire Sox $9^{+}$epithelium (Bankaitis et al. 2015). Together, these studies support the idea that low Neurog3 protein levels are functionally associated with promoting progenitormaintaining divisions, sustaining the actively cycling Neurog3 $3^{\text {TA.LO }}$ state (Fig. 7B). While reducing Neurog3 protein levels decreases endocrine biasing, particularly in the strong hypomorph context, its complete absence essentially totally ablates endocrine lineage potential /Gradwohl et al. 2000; Johansson et al. 2007; Wang et al. 2010). These observations indicate that the presence of low levels of Neurog3 protein is required to establish and maintain endocrine biasing, as opposed to commitment, in Sox $9^{+}$Neurog3 ${ }^{\text {TA }}$ cells.
Potentially important analogies can be drawn regarding these different behaviors driven by Neurog3 and the findings on how low versus high levels of Neurog2 maintain the cycling neural progenitor state or promote cell cycle exit and neural specification (Shimojo et al. 2008; Roybon et al. 2009; Ali et al. 2011; Florio et al. 2012). In those studies, Neurog $2^{\text {LO }}$ cells were proliferative, while homozygous Neurog2 inactivation stalled stem cell mitotic progression in the G1 phase, and overexpression accelerated cell cycle exit. In cycling progenitors, high Cdk activity and low Cdk inhibitor (Cdki) activity during G2-S-M phases keep Neurog2 in an unstable multiphosphorylated state subject to ubiquitylation-mediated degradation (Vosper et al. 2009; Ali et al. 2011). The low-level phospho-Neurog2 is unable to activate neural specification target genes but activates progenitor-associated targets (Ali et al. 2011; Hindley et al. 2012). In our case, the Sox $9^{+}$Neurog3 $3^{\text {TA.LO }}$ population comprises cells with low to undetectable Neurog3 protein, and future experimentation could test for Sox $9^{+}$Neurog3 ${ }^{\text {TA.LO }}$ progenitors containing low levels of an unstable phospho-Neurog3 protein that is periodically degraded during the cell cycle and is immunodetected, at least with current antibodies, in only a subset of the Neurog3-expressing Sox $9^{+}$endocrine-biased cells. Neurog3 has been shown to be inherently unstable and subject to the same ubiquitylation-mediated degradation mechanisms as Neurog2 (Roark et al. 2012; Qu et al. 2013). These striking parallels for Neurog2 and Neurog3 predict that low levels of unstable Neurog3 activate a subset of target genes specifically associated with promoting progenitor maintenance and the endocrine-biased state. The low level of Neurog3 expression in the endocrine progenitor context clearly has different consequences compared with the sustained low-level Neurog3 expression in adult $\beta$ cells that is necessary for physiological function: Neurog3 in mature $\beta$ cells most likely activates target genes peculiar to quiescent endocrine cells (Wang et al. 2009).

\section{Speculations on mechanisms regulating endocrine commitment vs. progenitor maintenance in Neurog $3^{\text {TA.LO }}$ progenitors}

Several studies show that the length of the G1 phase of the cell cycle can dictate the choice of progenitor maintenance versus lineage specification (Hindley et al. 2012; Pauklin and Vallier 2013; Hardwick et al. 2015). For Neurog2 in neural progenitors, the low Cdk/high Cdki activity and lengthened G1 keep Neurog2 nonphosphorylated/ underphosphorylated and stable enough to activate neuronal differentiation targets (Ali et al. 2011). Our model is that Neurog3 protein levels transition from lowsometimes immuno-undetectable but still present and functional-in cycling Neurog $3^{\text {TA.LO }}$ progenitors to uniformly high in endocrine-committing Neurog3 ${ }^{\text {TA.HI }}$ cells. Moreover, in every terminal differentiation division, we observed the irreversible transition to Neurog3 ${ }^{\text {TA.HI }}$ during what should be G1, 3-6 h after parental Neurog $3^{\text {TA.LO }}$ cell mitosis (Fig. 4; Supplemental Table S3). Moreover, enforcing G1-like conditions with the Cdki p27Kip1 led to significantly increased Neurog3 stability 
(Roark et al. 2012). In the Neurog3 ${ }^{\mathrm{HI}}$ state, Neurog3 promotes expression of the Cdki Cdkn1a (Miyatsuka et al. 2011), confirmed by our qRT-PCR analysis, creating a positive feed-forward loop driving cell cycle exit. If Neurog3 behaves like Neurog2, the G1 phase would be mechanistically coupled to Neurog3 accumulation for promoting the transition to endocrine lineage commitment.

We and others reported that altering Neurog3 expression, along with affecting endocrine specification commitment, disturbs the morphological development and maintenance of the epithelial endocrine niche environment (Magenheim et al. 2011; Bankaitis et al. 2015). Therefore, a new viewpoint is emerging - that a vital part of learning how to manipulate endocrine progenitor states (for example, toward sustained in vitro production of endocrine cells of specific fates) will be to understand how the endocrine niche environment integrates intercellular signaling and cell-autonomous programs to regulate specification and commitment. Since Neurog3 protein levels critically regulate progenitor versus endocrine commitment, we judged it important to determine how Neurog3 protein stability and accumulation are regulated in Sox $9^{+}$Neurog3 ${ }^{\text {TA }}$ progenitors. We were also interested in the possibility that intraepithelial Neurog3 expression within the Neurog3 $3^{\text {TA.LO }}$ population, similar to the Hes1-dependent oscillation of Neurog2 (Shimojo et al. 2008), undergoes dynamic oscillation between low-expressing and off states. Such oscillation might explain why Neurog3 protein falls below a detection threshold in many endocrine-biased progenitors as well as the cumulative labeling of the population using R26R-based lineage tracing. Investigating this issue would require novel Neurog3-driven reporters with the sensitivity to detect such dynamism. Characterizing these sorts of regulatory influences with increased spatiotemporal resolution could help to define how an endocrine specification commitment program is temporally deployed to generate the multilineage islets of Langerhans.

\section{Materials and methods}

Mice

Neurog3 ${ }^{\text {BAC.Cre }}[\mathrm{Tg}$ (Neurog3-cre)C1Able/J] (Schonhoff et al. 2004) and ROSA26R ${ }^{\text {EYFP }}\left[\mathrm{B} 6.129 \times 1-G t(R O S A) 26\right.$ Sor $\left.^{\text {tm1 } 1(E Y F P) C o s} / \mathrm{J}\right]$ (Srinivas et al. 2001) mice were described previously. Neurog3 $3^{\mathrm{EGFP} /+}$ [B6.129.S-Neurog3 ${ }^{\text {tm1(EGFP)Khk }} / \mathrm{Mmcd}$ (Lee et al. 2002) and Neurog3 ${ }^{\mathrm{FL} /+}$ [Neurog3 ${ }^{\text {tm1.1 (cre/ERT)Ggu }}$ (Wang et al. 2008) mice from Guoqiang Gu (Vanderbilt University) were described previously. All protocols were approved by the Vanderbilt University Institutional Animal Care and Use Committee. For genotyping primers, see Supplemental Table S5.

\section{Immunodetection}

Embryonic dorsal pancreata were fixed with $4 \%$ paraformaldehyde for $4-6 \mathrm{~h}$ at $4^{\circ} \mathrm{C}$. Cryosectioning was done on sucrose-equilibrated $\left(30 \%\right.$ overnight at $\left.4^{\circ} \mathrm{C}\right)$ OCT-embedded tissue (TissueTek). A Leica CM3050S was used to cut $10-\mu \mathrm{m}$ tissue sections, which were sequentially placed on three separate sets of slides, each covering $\sim 33 \%$ of the dorsal pancreas. For primary and secondary antibodies, see Supplemental Table S4.

\section{Flow sorting}

Multiple E12.5 or E14.5 Neurog $3^{\mathrm{RG} 1+}$ dorsal pancreata were pooled and treated with Accumax (Sigma) for 30-45 min (protocol available on request). Dispersed samples were washed and incubated on ice first with anti-Muc 1 antibody for $1 \mathrm{~h}$ and then antihamster Cy5 secondary antibody for an additional hour. Samples were washed in cold PBS, and DAPI was added to ensure sorting of viable cells. Flow sorting was performed with a BD FACSAria III.

\section{Static image acquisition and time-lapse movies}

Unless noted, images are epifluorescence from a Zeiss ApoTome microscope with Zeiss Axiovision software. All scale bars indicate $20 \mu \mathrm{m}$. Confocal immunofluorescence images (Fig. $1 \mathrm{H})$ and time-lapse movies were from a Zeiss LSM 510 confocal or LSM 780 two-photon microscope, respectively, analyzed with Zen 2.1 software. For time-lapse imaging, Neurog $3^{\text {RG1+ }}$ explants were embedded in BD Matrigel (BD Biosciences) and imaged immediately or allowed to attach to bovine fibronectin $(50 \mu \mathrm{g} / \mathrm{mL})$ overnight before imaging. All pancreatic explants were cultured in DMEM (without phenol-red) with $10 \%$ FBS, penicillin, and streptomycin $(10 \mathrm{U} / \mathrm{mL}$ each $)$ at $37^{\circ} \mathrm{C}$ in an environmentally controlled chamber.

\section{Acknowledgments}

We thank Richard Behringer (Baylor College of Medicine) for the $\mathrm{H}_{2} \mathrm{~B}^{\text {mCherry }}$-peptide-2A-GFP ${ }^{\mathrm{GPI}}$ reporter cassette. We thank Anna Means, Guoqiang Gu, Maureen Gannon, Roland Stein, Jake Kushner, and members of the Wright/Gu laboratories for discussions. This work used the Cell Imaging Shared Resource and Transgenic/Embryonic Stem Cell Shared Resource core facilities of the Vanderbilt Diabetes Research and Training Center funded by National Institute of Diabetes and Digestive and Kidney Diseases grant 020593. Flow cytometry was performed in the Vanderbilt University Medical Center Flow Cytometry Shared Resource supported by the Vanderbilt Ingram Cancer Center (P30 CA68485) and the Vanderbilt Digestive Disease Research Center (DK0558404). Generation of Neurog $3^{\mathrm{RG1}}$ mice was supported in part by the Beta Cell Biology Consortium Mouse Embryonic Stem Cell Core funded by the National Institute of Diabetes and Digestive and Kidney Diseases (U01DK072473). This study was supported by the National Institutes of Health/ National Institute of Diabetes and Digestive and Kidney Diseases (U01DK089570) and an American Heart Association fellowship to M.E.B. (13POST14240011).

\section{References}

Ali F, Hindley C, McDowell G, Deibler R, Jones A, Kirschner M, Guillemot F, Philpott A. 2011. Cell cycle-regulated multi-site phosphorylation of Neurogenin 2 coordinates cell cycling with differentiation during neurogenesis. Development 138: 4267-4277.

Bankaitis ED, Bechard ME, Wright CVE. 2015. Feedback control of growth, differentiation, and morphogenesis of pancreatic endocrine progenitors in an epithelial plexus niche. Genes Dev 29: 2203-2216.

Bradford JA, Clarke ST. 2011. Dual-pulse labeling using 5-ethynyl2'-deoxyuridine (Edu) and 5-bromo-2'-deoxyuridine (BrdU) in flow cytometry. Curr Protoc Cytom 55: 7.38.1-7.38.15.

Cano DA, Soria B, Martin F, Rojas A. 2014. Transcriptional control of mammalian pancreas organogenesis. Cell Mol Life Sci 71: 2383-2402. 
Desgraz R, Herrera PL. 2009. Pancreatic neurogenin 3-expressing cells are unipotent islet precursors. Development 136: $3567-$ 3574.

De Vas MG, Kopp JL, Heliot C, Sander M, Cereghini S, Haumaitre C. 2015. Hnf1b controls pancreas morphogenesis and the generation of $\mathrm{Ngn}^{+}$endocrine progenitors. Development 142: 871-882.

Ejarque M, Cervantes S, Pujadas G, Tutusaus A, Sanchez L, Gasa R. 2013. Neurogenin 3 cooperates with Foxa2 to autoactivate its own expression. J Biol Chem 288: 11705-11717.

Florio M, Leto K, Muzio L, Tinterri A, Badaloni A, Croci L, Zordan P, Barili V, Albieri I, Guillemot F, et al. 2012. Neurogenin 2 regulates progenitor cell-cycle progression and Purkinje cell dendritogenesis in cerebellar development. Development 139: 2308-2320.

Gradwohl G, Dierich A, LeMeur M, Guillemot F. 2000. Neurogenin3 is required for the development of the four endocrine cell lineages of the pancreas. Proc Natl Acad Sci 97: 16071611.

Gu G, Dubauskaite J, Melton DA. 2002. Direct evidence for the pancreatic lineage: $\mathrm{NGN}^{+}$cells are islet progenitors and are distinct from duct progenitors. Development 129: 2447-2457.

Hardwick LJA, Ali FR, Azzarelli R, Philpott A. 2015. Cell cycle regulation of proliferation versus differentiation in the central nervous system. Cell Tissue Res 359: 187-200.

Hindley C, Ali F, McDowell G, Cheng K, Jones A, Guillemot F, Philpott A. 2012. Post-translational modification of Ngn2 differentially affects transcription of distinct targets to regulate the balance between progenitor maintenance and differentiation. Development 139: 1718-1723.

Johansson KA, Dursun U, Jordan N, Gu G, Beermann F, Gradwohl G, Grapin-Botton A. 2007. Temporal control of neurogenin3 activity in pancreas progenitors reveals competence windows for the generation of different endocrine cell types. Dev Cell 12: 457-465.

Kim YH, Larsen HL, Rué P, Lemaire LA, Ferrer J, Grapin-Botton A. 2015. Cell cycle-dependent differentiation dynamics balances growth and endocrine differentiation in the pancreas. PLOS Biol 13: e1002111.

Lee JC, Smith SB, Watada H, Lin J, Scheel D, Wang J, Mirmira RG, German MS. 2001. Regulation of the pancreatic pro-endocrine gene neurogenin3. Diabetes 50: 928-936.

Lee CS, Perreault N, Brestelli JE, Kaestner KH. 2002. Neurogenin 3 is essential for the proper specification of gastric enteroendocrine cells and the maintenance of gastric epithelial cell identity. Genes Dev 16: 1488-1497.

Magenheim J, Klein AM, Stanger BZ, Ashery-Padan R, SosaPineda B, Gu G, Dor Y. 2011. Ngn $3^{+}$endocrine progenitor cells control the fate and morphogenesis of pancreatic ductal epithelium. Dev Biol 359: 26-36.

Miyatsuka T, Kosaka Y, Kim H, German MS. 2011. Neurogenin3 inhibits proliferation in endocrine progenitors by inducing Cdknla. Proc Natl Acad Sci 108: 185-190.

Nimmo RA, May GE, Enver T. 2015. Primed and ready: understanding lineage commitment through single cell analysis. Trends Cell Biol 25: 459-467.

Pan FC, Wright C. 2011. Pancreas organogenesis: from bud to plexus to gland. Dev Dyn 240: 530-565.

Pauklin S, Vallier L. 2013. The cell-cycle state of stem cells determines cell fate propensity. Cell 155: 135-147.

Puri S, Hebrok M. 2007. Dynamics of embryonic pancreas development using real-time imaging. Dev Biol 306: 82-93.

Qu X, Afelik S, Jensen JN, Bukys MA, Kobberup S, Schmerr M, Xiao F, Nyeng P, Veronica Albertoni M, Grapin-Botton A, et al. 2013. Notch-mediated post-translational control of
Ngn3 protein stability regulates pancreatic patterning and cell fate commitment. Dev Biol 376: 1-12.

Roark R, Itzhaki L, Philpott A. 2012. Complex regulation controls Neurogenin3 proteolysis. Biol Open 1: 1264-1272.

Roybon L, Hjalt T, Stott S, Guillemot F, Li J-Y, Brundin P. 2009. Neurogenin 2 directs granule neuroblast production and amplification while NeuroD1 specifies neuronal fate during hippocampal neurogenesis. PLoS One 4: e4779.

Schonhoff SE, Giel-Moloney M, Leiter AB. 2004. Neurogenin 3expressing progenitor cells in the gastrointestinal tract differentiate into both endocrine and non-endocrine cell types. Dev Biol 270: 443-454.

Seymour PA, Freude KK, Tran MN, Mayes EE, Jensen J, Kist R, Scherer G, Sander M. 2007. SOX9 is required for maintenance of the pancreatic progenitor cell pool. Proc Natl Acad Sci 104: 1865-1870.

Shaner NC, Steinbach PA, Tsien RY. 2005. A guide to choosing fluorescent proteins. Nat Methods 2: 905-909.

Shimojo H, Ohtsuka T, Kageyama R. 2008. Oscillations in notch signaling regulate maintenance of neural progenitors. Neuron 58: $52-64$.

Solar M, Cardalda C, Houbracken I, Martín M, Maestro MA, De Medts N, Xu X, Grau V, Heimberg H, Bouwens L, et al. 2009. Pancreatic exocrine duct cells give rise to insulin-producing $\beta$ cells during embryogenesis but not after birth. Dev Cell 17: 849-860.

Srinivas S, Watanabe T, Lin CS, William CM, Tanabe Y, Jessell TM, Costantini F. 2001. Cre reporter strains produced by targeted insertion of EYFP and ECFP into the ROSA26 locus. BMC Dev Biol 1: 4.

Stephens DJ, Allan VJ. 2003. Light microscopy techniques for live cell imaging. Science 300: 82-86.

Teta M, Rankin MM, Long SY, Stein GM, Kushner JA. 2007. Growth and regeneration of adult $\beta$ cells does not involve specialized progenitors. Dev Cell 12: 817-826.

Villasenor A, Chong DC, Cleaver O. 2008. Biphasic Ngn3 expression in the developing pancreas. Dev Dyn 237: 3270-3279.

Vosper JMD, McDowell GS, Hindley CJ, Fiore-Heriche CS, Kucerova R, Horan I, Philpott A. 2009. Ubiquitylation on canonical and non-canonical sites targets the transciption factor neurogenin for ubiquitin-mediated proteolysis. I Biol Chem 284: $15458-15468$.

Wang S, Hecksher-Sorensen J, Xu Y, Zhao A, Dor Y, Rosenberg L, Serup P, Gu G. 2008. Myt1 and Ngn3 form a feed-forward expression loop to promote endocrine islet cell differentiation. Dev Biol 317: 531-540.

Wang S, Jensen JN, Seymour PA, Hsu W, Dor Y, Sander M, Magnuson MA, Serup P, Gu G. 2009. Sustained Neurog3 expression in hormone-expressing islet cells is required for endocrine maturation and function. Proc Natl Acad Sci 106: 9715-9720.

Wang S, Yan J, Anderson DA, Xu Y, Kanal MC, Cao Z, Wright CVE, Gu G. 2010. Neurog3 gene dosage regulates allocation of endocrine and exocrine cell fates in the developing mouse pancreas. Dev Biol 339: 26-37.

Wilkinson G, Dennis D, Schuurmans C. 2013. Proneural genes in neocortical development. Neuroscience 253: 256-273.

Zhang H, Tweedie E, Pope CF, Washington MK, Hipkens S, Means AL, Path G, Seufert J, Costa RH, Leiter AB, et al. 2009. Multiple, temporal-specific roles for HNF6 in pancreatic endocrine and ductal differentiation. Mech Dev 126: 958-973.

Zhou Q, Law AC, Rajagopal J, Anderson WJ, Gray PA, Melton DA. 2007. A multipotent progenitor domain guides pancreatic organogenesis. Dev Cell 13: 103-114. 


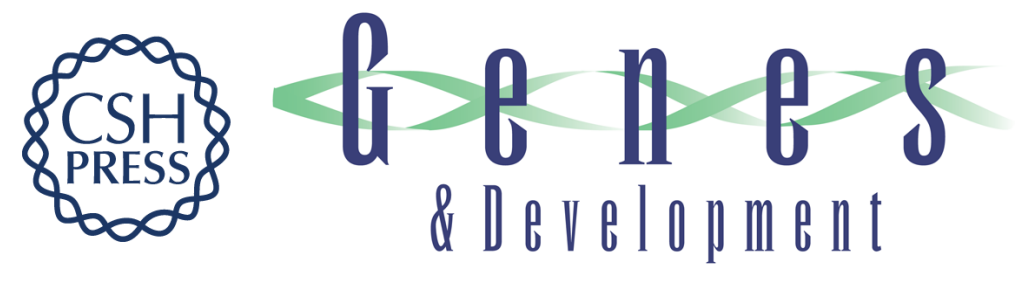

\title{
Precommitment low-level Neurog3 expression defines a long-lived mitotic endocrine-biased progenitor pool that drives production of endocrine-committed cells
}

\author{
Matthew E. Bechard, Eric D. Bankaitis, Susan B. Hipkens, et al.
}

Genes Dev. 2016, 30: originally published online September 1, 2016

Access the most recent version at doi:10.1101/gad.284729.116

Supplemental Material

References

Creative

Commons

License

Email Alerting

Service
http://genesdev.cshlp.org/content/suppl/2016/08/31/gad.284729.116.DC1

This article cites 41 articles, 16 of which can be accessed free at: http://genesdev.cshlp.org/content/30/16/1852.full.html\#ref-list-1

This article is distributed exclusively by Cold Spring Harbor Laboratory Press for the first six months after the full-issue publication date (see http://genesdev.cshlp.org/site/misc/terms.xhtml). After six months, it is available under a Creative Commons License (Attribution-NonCommercial 4.0 International), as described at http://creativecommons.org/licenses/by-nc/4.0/.

Receive free email alerts when new articles cite this article - sign up in the box at the top right corner of the article or click here.

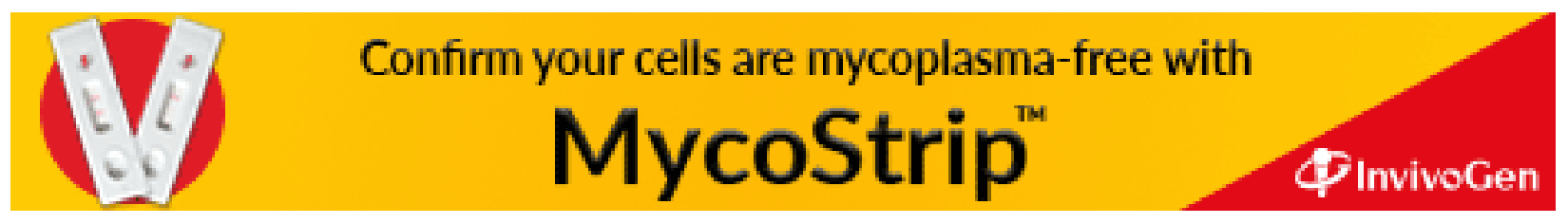

